\title{
Infinite Tensor Products of Operators
}

\author{
By \\ Yoshiomi NAKAGAMI*
}

\section{§1. Introduction}

In the previous paper [8] we established a definition of an infinite tensor product $\otimes x_{\iota}$ of operators on $\otimes \mathscr{H}_{\iota}$ and studied its properties under the assumption: $\Pi\left\|x_{\imath}\right\|<+\infty$. In some applications, for instance, to Tomita's theory [3] and to quantum field theory $[13,14]$, we are obliged to work with a weaker assumption.

In the present paper, we shall define an infinite tensor product $\otimes c^{\prime} c x_{\iota}$ of operators $x_{\iota}$ on $\mathscr{H}_{\iota}$ as a closed linear mapping from an incomplete infinite tensor product space $\otimes^{c} \mathscr{H}_{\iota}$ to another $\otimes^{c^{\prime}} \mathscr{H}_{\iota}$. We do not make any assumption on $\left\|x_{\imath}\right\|$, allowing unbounded closed operators $x_{\iota}$. The crucial assumption on $\left(x_{\iota}\right)$ is the existence of what we call a non-zero reference pair. This assumption turns out to be sufficiently general to allow various applications and yet sufficiently strong to yield significant results. Typical result is the following:

Theorem 1.1. If $x_{\iota}$ is positive self-aljoint and $\left(\xi_{0 \iota}, \eta_{0_{\iota}}\right)$ is a nonzero reference pair of $\left(x_{\iota}\right)$, then $\left(\xi_{0 \iota}\right)$ and $\left(\eta_{0 \iota}\right)$ belong to the same equivalence class $c$ and $\otimes c^{c} x_{\iota}$ is essentially self-adjoint on the linear span of the product vectors $\otimes \xi_{\iota}$ such that $\xi_{\iota}=\xi_{0}$ except for a finite number of $\iota$ and $\xi_{\iota}$ is in the domain of $x_{\iota}$.

Terminologies here are defined as follows:

Definition 1.1. A pair $\left(\xi_{0_{\iota}}, \eta_{0_{\iota}}\right)$ is a non-zero reference pair of $\left(x_{\iota}\right)$ if the following conditions are fulfilled:

(a) $\left(\xi_{0 \iota}\right)$ and $\left(\eta_{0 \iota}\right)$ are $C_{0}$-sequences;

Communicated by H. Araki, October 19, 1973.

* Department of Mathematics, Tokyo Institute of Technology, Tokyo. 


$$
\xi_{0 \iota} \neq 0, \sum\left|\left\|\xi_{0 \iota}\right\|^{2}-1\right|<+\infty, \eta_{0 \iota} \neq 0, \sum\left|\left\|\eta_{0 \iota}\right\|^{2}-1\right|<+\infty .
$$

(b) $\xi_{0 \iota}$ is in the domain of $x_{\iota}$ and $\left(x_{\iota} \xi_{0 \iota}\right)$ is a $C$-sequence;

$$
\sum\left|\left\|x_{\iota} \xi_{0 \iota}\right\|^{2}-1\right|<+\infty
$$

(c) $\left(x_{\iota} \xi_{0 \iota}\right)$ is equivalent to $\left(\eta_{0_{\iota}}\right)$;

$$
\sum\left|\left(x_{\iota} \xi_{0 \iota} \mid \eta_{0 \iota}\right)-1\right|<+\infty
$$

(d) $\eta_{0 \iota}$ is in the domain of $x_{\iota}^{*}$ and $\left(x_{\iota}^{*} \eta_{0 \iota}\right)$ is a $C$-sequence;

$$
\sum\left|\left\|x_{\iota}^{*} \eta_{0 \iota}\right\|^{2}-1\right|<+\infty
$$

All assumptions except for (d) are obviously unavoidable if we want to define what can be denoted by $\otimes^{c^{\prime} c} x_{\iota}$. The assumption (d) is crucial and enables all calculations go through.

The product operators $\otimes c^{\prime} c x_{\iota}$ for $c \equiv c\left(\xi_{0 \iota}\right)$ and $c^{\prime} \equiv c\left(x_{\iota} \xi_{0 \iota}\right)$ is defined in three steps: On the product vector $\otimes \xi_{\iota}$ with $\xi_{\iota}=\xi_{0 \iota}$ except for a finite number of $\iota$ and $\xi_{\iota}$ in the domain of $x_{\iota}$, a mapping $\odot\left(x_{\iota}\right.$, $\left.\xi_{0 \iota}\right)$ is defined by

$$
\odot\left(x_{\iota}, \xi_{0 \iota}\right) \otimes \xi_{\iota}=\otimes x_{\iota} \xi_{\iota} .
$$

It is then proved to be extendable linearly to the linear span of such product vectors (denoted as $\left.\odot\left(D\left(x_{\iota}\right), \xi_{0}\right)\right)$. It is then proved to be closable and the closure is denoted by $\otimes{ }^{\prime} c x_{\iota}$. The assumption (d) is necessary for this closability (Remark 2.2).

All these discussions and the proof of the formula

$$
\left(\otimes c^{\prime} c x_{\iota}\right)^{*}=\otimes{ }^{c c^{\prime}} x_{\iota}^{*}
$$

are given in Section 2. This formula contains Theorem 1.1 as a special case $x_{\imath}^{*}=x_{\iota}$.

In Section 3, we give several conditions for the existence of a non-zero reference pair, one of which is closely related to Kolmogorov's three series theorem. Theorem 3.1 has a close connection with some results of Reed [13] and Streit [14].

In Section 4, we apply our result to a modular operators $\Delta_{\xi}$, and show that $\otimes^{c} \Delta_{\xi}$, is a modular operator for $\otimes \xi_{\text {c }}$ where $\left(\xi_{\iota}\right) \in c$. 
In Section 5, we apply our results to an infinite product $\mu$ of $\sigma$ finite measures $\mu_{\imath}$. Theorem in Section 3 gives us conditions for the equivalence $\mu \sim v$ when $\mu_{\iota}$ is equivalent to a given probability measure $v_{\iota}$ and $v$ is the product measure of $v_{\iota}$. One of the conditions reproduces a result of Hill [5].

The discussion in Section 5 is generalized to an infinite product of semi-finite faithful normal weights in Section 6. The result is used in a separate paper [6].

Notations: For standard definitions and notations for infinite tensor products of Hilbert spaces and von Neumann algebras, see [11]. Let $I$ be an infinite index set and $J \subset \subset I$ indicates that $J$ is a finite subset of $I . S$ denotes the set of all $C$-sequences $\left(\xi_{\iota}\right)$ (i.e., $\sum\left|\left\|\xi_{\iota}\right\|^{2}-1\right|<+\infty$ ) and $S_{0}$ denotes the set of all $C_{0}$-sequences $\left(\xi_{\iota}\right)$ (i.e., $\left(\xi_{\iota}\right) \in S, \xi_{\iota} \neq 0$ ). The word "sequence" is used for $\left(\xi_{\iota}\right)$ even if $I$ is uncountably infinite. $\left(\xi_{\iota}\right) \sim\left(\eta_{\iota}\right)$ denotes the condition $\sum\left|\left(\xi_{\iota} \mid \eta_{\iota}\right)-1\right|<+\infty$. It defines equivalence relations in $S$ and in $S_{0}$. The equivalence class of $\left(\xi_{\iota}\right)$ is denoted by $c\left(\xi_{\iota}\right)$. The incomplete infinite tensor product $\mathscr{H}_{c} \equiv \otimes^{c} \mathscr{H}_{\iota}$ is spanned by

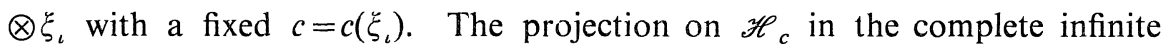
tensor product $\otimes \mathscr{H}_{\iota}$ is denoted by $p_{c}$. Let $\left(\xi_{\iota}\right),\left(\eta_{\iota}\right) \in S$ and $c \equiv c\left(\xi_{\iota}\right)$, $c^{\prime} \equiv c\left(\eta_{\iota}\right) . \quad\left(\xi_{\iota}\right) \quad$ and $\quad c$ are $u$-equivalent (resp. $p$-equivalent) to $\left(\eta_{\iota}\right)$ and $c^{\prime}$, respectively, if $\left(\xi_{\iota}\right) \sim\left(u_{\iota} \eta_{\iota}\right)$ for some unitary (resp. partial isometry) $u_{\iota} \in M_{\iota}^{\prime}$. This is denoted by $\left(\xi_{\iota}\right) \underset{u}{\sim}\left(\eta_{\iota}\right), \underset{u}{c \sim c^{\prime}}\left(\operatorname{resp} .\left(\xi_{\iota}\right) \underset{p}{\sim}\left(\eta_{\iota}\right), \underset{p}{c \sim c^{\prime}}\right)$. If $I$ is countable, $\underset{p}{c \sim c^{\prime}}$ and $c \underset{u}{\sim} c^{\prime}$ are equivalent, [1]. Let $p(c)$ denote the central carrier of $p_{c}$ in $\left(\otimes M_{\iota}\right)^{\prime} . \quad p(c)$ is the sum of $p_{c^{\prime}}$ with $c^{\prime} \underset{p}{\sim}$, [1]. For $x_{\iota} \in B\left(\mathscr{H}_{\iota}\right)$ with $\Pi\left\|x_{\imath}\right\|<+\infty$, we can define an infinite tensor product $\otimes x_{\iota}$ of operators, which is bounded on $\otimes \mathscr{H}_{\iota}$. When $\mathscr{H}_{c}$ is invariant under $\otimes x_{\iota}$, the induction of $\otimes x_{\iota}$ to $\mathscr{H}_{c}$ is denoted by $\otimes^{c} x_{\iota}$ or $\left(\otimes_{J} x_{\iota}\right) \otimes\left(\otimes_{I \backslash J}^{c} x_{\iota}\right)$ for $J \subset \subset I$.

\section{§2. Infinite Tensor Products of Operators}

For an operator $x$ (resp. $y$ ) with domain $D(x)$ (resp. $D(y)$ ), let $D(x) \odot$ $D(y)$ denote the algebraic tensor product in $\overline{D(x)} \otimes \overline{D(y)}$ of $D(x)$ and $D(y)$, and $x \odot y$ the operator on $D(x) \odot D(y)$ defined by 
for all $\xi \in D(x)$ and $\eta \in D(y)$.

Lemma 2.1. If $x$ and $y$ are essentially self-adjoint, then $x \odot y$ and $\bar{x} \odot \bar{y}$ are essentially self-adjoint and $\overline{x \odot y}=\overline{\bar{x} \odot \bar{y}}$.

For self-adjoint operators $x$ and $y$, we denote $\overline{x \odot y}$ by $x \otimes y$ in the following.

Throughout this and next sections $x_{\iota}$ is a non zero densely defined closed operator on a Hilbert space $\mathscr{H}_{\iota}, x_{\iota}=u_{\imath}\left|x_{\iota}\right|$ is the polar decomposition of $x_{\iota}$, and $D\left(x_{\iota}\right)$ denotes the domain of $x_{\iota}$.

For $\left(\xi_{0_{\iota}}\right) \in S_{0}$ with $\xi_{0 \iota} \in D\left(x_{\iota}\right)$ and $\left(x_{\iota} \xi_{0 \iota}\right) \in S$, we denote by $\odot\left(D\left(x_{\iota}\right)\right.$, $\xi_{0 \iota}$ ) the linear span of $\otimes \xi_{\iota}$ such that $\xi_{\iota}=\xi_{0 \iota}$ for all but a finite number of $\iota \in I$ and $\xi_{\iota} \in D\left(x_{\imath}\right)$ for all $\iota \in I$.

Lemma 2.2. Let $\left(\xi_{0 \iota}\right) \in S_{0}$ and $\xi_{0 \iota} \in D\left(x_{\iota}\right)$ for all $\iota \in I$. If $\left(x_{\iota} \xi_{0 \iota}\right) \in$ $S$, there exists a non zero operator $x$ with domain $\odot\left(D\left(x_{\iota}\right), \xi_{0}\right)$ such that $x \otimes \xi_{\iota}=\otimes x_{\iota} \xi_{\iota}$ for all $\otimes \xi_{\iota}$ in $\odot\left(D\left(x_{\iota}\right), \xi_{0 \iota}\right)$.

Proof. For $\xi=\sum_{k=1}^{n} \otimes \xi_{k \iota}$ in $\odot\left(D\left(x_{\iota}\right), \xi_{0 \iota}\right)$, there exists a $J \subset \subset I$ such that $\xi=\xi_{J} \otimes\left(\otimes_{I \backslash J} \xi_{0 \iota}\right)$ for $\xi_{J} \in \otimes_{J} \mathscr{H}_{\iota}$ and $\xi_{J}=\sum_{k=1}^{n} \otimes_{J} \xi_{k_{\iota}}$. Since $\left(\xi_{0}\right) \in S_{0}$, if $\xi=0$ then $\xi_{J}=0$ and so $\left(\otimes_{J} x_{\iota}\right) \xi_{J}=0$. Therefore $\sum_{k=1}^{n}$ $\otimes x_{\iota} \xi_{k \iota}=0$. Thus the mapping

$$
\sum_{k=1}^{n} \otimes \xi_{k \iota} \longmapsto \sum_{k=1}^{n} \otimes x_{\imath} \xi_{k \iota}
$$

is well defined. We denote it by $x$. Since $\left(x_{\iota} \xi_{0 \iota}\right) \in S$, there exists a $\otimes \xi_{\iota}$ in $\odot\left(D\left(x_{\iota}\right), \xi_{0_{\iota}}\right)$ with $\left(x_{\imath} \xi_{\iota}\right) \in S_{0}$. Therefore $x$ is non zero.

Q.E.D.

Definition 2.1. Let $\left(\xi_{0 \iota}\right) \in S_{0}$ and $\Pi\left\|x_{\iota} \xi_{0 \iota}\right\|<+\infty$. An operator $\odot\left(x_{\iota}, \xi_{0 \iota}\right)$ on $\odot\left(D\left(x_{\iota}\right), \xi_{0 \iota}\right)$ is defined by

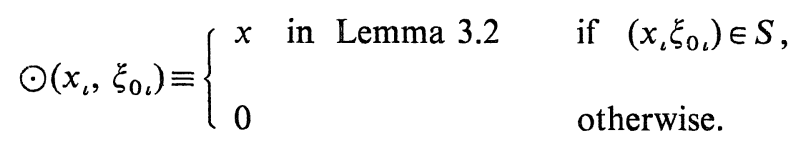


The following lemma is immediate from Definition 1.1 .

Lemma 2.3. The following three conditions are equivalent:

(i) $\left(\xi_{0}, \eta_{0_{\iota}}\right)$ is a non-zero reference pair of $\left(x_{\iota}\right)$;

(ii) $\xi_{0 \iota} \in D\left(x_{\iota}\right), \eta_{0 \iota} \in D\left(x_{\iota}^{*}\right),\left(\xi_{0 \iota}\right) \in S_{0},\left(\eta_{u_{\iota}}\right) \in S_{0},\left(x_{\iota} \xi_{0 \iota}\right) \in S, \quad\left(x_{\iota}^{*} \eta_{0 \iota}\right) \in S$ and $\left(x_{\iota} \xi_{0 \iota}\right) \sim\left(\eta_{0 \iota}\right)$; and

(iii) $\left(\eta_{0_{\iota}}, \xi_{0_{\iota}}\right)$ is a non-zero reference pair of $\left(x_{\iota}^{*}\right)$.

Example 2.1. For $0<\varepsilon_{\iota}<1, \iota \in I$, put

$$
x_{\iota} \equiv\left(\begin{array}{cc}
\varepsilon_{\iota}^{-1} & 0 \\
0 & 1
\end{array}\right) \text { and } \xi_{\iota} \equiv \eta_{\iota} \equiv\left(\begin{array}{c}
\varepsilon_{\iota}^{2} \\
1
\end{array}\right) \text {. }
$$

If $\sum \varepsilon_{\iota}^{2}<+\infty$, then $x_{\iota}>0,\left(\xi_{\iota}\right)=\left(\eta_{\iota}\right) \in S_{0},\left(x_{\iota} \xi_{\iota}\right)=\left(x_{\iota} \eta_{\iota}\right) \in S_{0}$ and $\left(x_{\iota} \xi_{\iota}\right) \sim$ $\left(\eta_{\iota}\right)$. But $\left(x_{\iota}^{2} \xi_{\iota}\right) \notin S$.

Lemma 2.4. If $\left(\xi_{0_{\iota}}, \eta_{0_{\iota}}\right)$ is a non-zero reference pair of $\left(x_{\iota}\right)$, then

(i) $\odot\left(x_{\iota}, \xi_{0_{\iota}}\right) \odot\left(D\left(x_{\iota}\right), \xi_{0_{\iota}}\right) \subset \mathscr{H}_{c^{\prime}}$ for $c^{\prime} \equiv c\left(\eta_{0_{\iota}}\right)$;

(ii) $\left(\odot\left(x_{\iota}, \xi_{0_{\iota}}\right)\right)^{*} \supset \odot\left(x_{\iota}^{*}, \eta_{0 \iota}\right)$ and $\odot\left(x_{\iota}, \xi_{0_{\iota}}\right)$ is closable; and

(iii) for the closure $x$ of $\odot\left(x_{\iota}, \xi_{0_{\iota}}\right), x^{*} x$ is a self-adjoint operator on $\mathscr{H}_{c}$ for $c \equiv c\left(\xi_{0_{\iota}}\right)$.

Proof. (i) It is clear from Lemma 2.2 .

(ii) For all $\otimes \xi_{\iota} \in \odot\left(D\left(x_{\iota}\right), \xi_{0 \iota}\right)$ and $\otimes \eta_{\iota} \in \odot\left(D\left(x_{\iota}^{*}\right), \eta_{0_{\iota}}\right)$ we have

$$
\begin{aligned}
& \left(\odot\left(x_{\iota}, \xi_{0}\right) \otimes \check{\zeta}_{\iota} \mid \otimes \eta_{\iota}\right)=\left(\otimes x_{\iota} \xi_{\iota} \mid \otimes \eta_{\iota}\right) \\
& \quad=\Pi\left(x_{\iota} \xi_{\iota} \mid \eta_{\iota}\right)=\Pi\left(\xi_{l} \mid x_{\iota}^{*} \eta_{\iota}\right) \\
& \quad=\left(\otimes \ddot{\zeta}_{\iota} \mid \otimes x_{\iota}^{*} \eta_{\iota}\right)=\left(\otimes \xi_{\iota} \mid \odot\left(x_{\iota}^{*}, \eta_{0 \iota}\right) \otimes \eta_{\iota}\right) .
\end{aligned}
$$

Since $\odot\left(D\left(x_{\iota}\right), \xi_{0_{\iota}}\right)$ is dense in $\mathscr{H}_{c}$ for $c \equiv c\left(\xi_{0_{\iota}}\right)$ and $\odot\left(D\left(x_{\iota}^{*}\right), \eta_{0_{\iota}}\right)$ is dense in $\mathscr{H}_{c^{\prime}}$ for $c^{\prime} \equiv c\left(\eta_{0_{\iota}}\right)$, it follows that $\odot\left(x_{\iota}^{*}, \eta_{0_{\iota}}\right) \subset\left(\odot\left(x_{\iota}, \xi_{0_{\iota}}\right)\right)^{*}$.

(iii) Since $x$ is a closed operator of $\mathscr{H}_{c}$ to $\mathscr{H}_{c^{\prime}}, x^{*}$ is an operator of $\mathscr{H}_{c^{\prime}}$ to $\mathscr{H}_{c}$. Therefore $x^{*} x$ is self-adjoint on $\mathscr{H}_{c}$.

Q.E.D.

Lemma 2.5. Let $\left(\xi_{j \iota}, \eta_{j \iota}\right)$ be a non-zero reference pair of $\left(x_{\iota}\right)$ for $j=0,1$. If $c\left(\xi_{0_{\iota}}\right)=c\left(\xi_{1_{\imath}}\right)$, then $c\left(\eta_{0_{\iota}}\right)=c\left(\eta_{1_{\imath}}\right)$ and the closure of $\odot\left(x_{1}\right.$, $\left.\xi_{0_{\iota}}\right)$ is the closure of $\odot\left(x_{\iota}, \xi_{1 \iota}\right)$. 
Proof. Since $\left(\xi_{j \iota}, \eta_{j_{\iota}}\right)$ is a non-zero reference pair of $\left(x_{\iota}\right)$, we have $\left(x_{\iota} \xi_{0 \iota}\right) \sim\left(\eta_{0 \iota}\right)$ and $\left(\xi_{1 \iota}\right) \sim\left(x_{\iota}^{*} \eta_{1} \iota\right)$. Since $\left(\xi_{0 \iota}\right) \sim\left(\xi_{1 \iota}\right)$ by assumption, we have $\left(\xi_{0 \iota}\right) \sim\left(x_{\iota}^{*} \eta_{1 \iota}\right)$ and hence $\left(x_{\iota} \xi_{0 \iota}\right) \sim\left(\eta_{1}\right)$. Therefore $\left(\eta_{0_{\iota}}\right) \sim\left(\eta_{1_{\iota}}\right)$. Let $c \equiv c\left(\xi_{0_{\iota}}\right)$ and $c^{\prime} \equiv c\left(\eta_{0_{\iota}}\right)$. Since $\left(\xi_{0 \iota}\right) \sim\left(\xi_{1 \iota}\right)$ and $\left(x_{\iota} \xi_{0 \iota}\right) \sim\left(x_{\iota} \xi_{1 \iota}\right)$, there exists for any $\varepsilon>0$ a $J_{1} \subset \subset I$ such that

$$
\left\|\otimes \xi_{1 \iota}-\left(\underset{J}{\otimes} \xi_{1 \iota}\right) \otimes\left(\underset{I \backslash J}{\otimes} \xi_{0 \iota}\right)\right\|<\varepsilon
$$

and

$$
\left.\| \otimes x_{\iota} \xi_{1 \iota}-\left(\underset{J}{\otimes} x_{\iota} \xi_{1 \iota}\right) \otimes \underset{I \backslash J}{\otimes} x_{\iota} \xi_{0 \iota}\right) \|<\varepsilon
$$

for all $J_{1} \subset J \subset \subset I$. Therefore $\otimes \xi_{1 \iota}$ is in the domain of $\overline{\odot\left(x_{\iota}, \xi_{0 \iota}\right)}$ and hence $\overline{\odot\left(x_{\iota}, \xi_{1 \iota}\right)} \subset \overline{\odot\left(x_{\iota}, \xi_{0}\right)}$. The converse inclusion is proved similarly.

Q.E.D.

Definition 2.2. The closed operator in Lemma 2.4 is denoted by $\otimes^{c^{c}} x_{\imath} . \otimes^{c c} x_{\iota}$ is also denoted by $\otimes^{c} x_{\iota}$.

For a non-zero reference pair $\left(\xi_{0 \iota}, \eta_{0_{\iota}}\right)$ of $\left(x_{\iota}\right)$ if $\left(\xi_{\iota}\right) \in S_{0}$ with $\otimes \xi_{\iota} \in \odot\left(D\left(x_{\iota}\right), \xi_{0_{\iota}}\right)$ and if $\left(\eta_{\iota}\right) \in S_{0}$ with $\otimes \eta_{\iota} \in \odot\left(D\left(x_{\iota}^{*}\right), \eta_{0_{\iota}}\right)$, then $\left(\xi_{\iota}, \eta_{\iota}\right)$ is a non-zero reference pair of $\left(x_{\iota}\right)$.

We are now ready to prove the main theorem.

Proof of Theorem 1.1. Let $s\left(x_{\iota}\right)$ be the carrier projection of $x_{\iota}$. Since $\left(\xi_{0_{\iota}}\right) \in S,\left(x_{\iota}^{*} \eta_{0_{\iota}}\right) \in S,\left\|s\left(x_{\iota}\right)\right\|=1$ and $\left(s\left(x_{\iota}\right) \xi_{0 \iota}\right) \sim\left(x_{\iota}^{*} \eta_{0_{\iota}}\right)$, it follows from Lemma 1 in [1] that $\left(s\left(x_{\iota}\right) \xi_{0 \iota}\right) \in S$. Therefore there is a $\left(\xi_{\iota}\right) \in S_{0}$ such that $\otimes \xi_{\iota} \in \odot\left(D\left(x_{\iota}\right), \xi_{0 \iota}\right)$ and $\otimes s\left(x_{\iota}\right) \xi_{\iota} \neq 0$. Since $\left(\xi_{\iota}, \eta_{0 \iota}\right)$ is a nonzero reference pair of $\left(x_{\iota}\right)$ and $\odot\left(x_{\iota}, \xi_{0 \iota}\right)=\odot\left(x_{\iota}, \xi_{\iota}\right)$, we may assume that $\otimes s\left(x_{\iota}\right) \xi_{0 \iota} \neq 0$ by choosing such a $\left(\xi_{\iota}\right)$ as $\left(\xi_{0 \iota}\right)$.

Let $x$ denote the operator $\otimes c^{\prime} c x_{\iota}$ for $c \equiv c\left(\xi_{0 \iota}\right)$ and $c^{\prime} \equiv c\left(\eta_{0_{\iota}}\right)$. Let

$$
\underset{I \backslash J}{\otimes c^{\prime} c} x_{\iota}=u(I \backslash J) y(I \backslash J)
$$

be the polar decomposition of $\otimes_{I}^{c^{\prime} c_{J}} x_{\iota}$ for any $J \subset \subset I$. Put $y_{J} \equiv\left(\otimes_{J} x_{\iota}\right)$ $\otimes y(I \backslash J)$. Since $y_{J}$ is positive self-adjoint on $\mathscr{H}_{c}$,

$$
|x|=\left(x^{*} x\right)^{1 / 2}=\left(y_{J}^{*} y_{J}\right)^{1 / 2}=y_{J} .
$$


Putting $u \equiv u(I)$ and $u_{J} \equiv\left(\otimes_{J} s\left(x_{\iota}\right)\right) \otimes u(I \backslash J)$, we have

$$
\begin{aligned}
u|x| & =\otimes{ }^{c^{\prime} c} x_{\iota}=\left(\underset{J}{\otimes} x_{\iota}\right) \otimes \underset{I \backslash J}{\otimes} \underbrace{c^{\prime} c} x_{\iota}) \\
& =\left(\bigotimes_{J} x_{\iota}\right) \otimes u(I \backslash J) y(I \backslash J)=u_{J} y_{J} .
\end{aligned}
$$

The uniqueness of a polar decomposition implies that $u=u_{J}$ and $u$ transforms $\mathscr{H}_{c}$ to $\mathscr{H}_{c^{\prime}}$. Since $u_{J}=u \neq 0$, we have $u(I \backslash J)\left(\otimes_{I \backslash J} \xi_{0_{\iota}}\right) \neq 0$ for some $J$. Since $\left(s\left(x_{\iota}\right) \xi_{0_{\iota}}\right) \in S_{0}$, we have $u \otimes \xi_{0 \iota} \neq 0$. Accordingly there exists a $\left(\zeta_{\iota}\right) \in S$ and a $\zeta \in \mathscr{H}_{c^{\prime}}$ such that $\left\|\zeta_{\iota}\right\|=1, c\left(\zeta_{\iota}\right)=c^{\prime},\left(\zeta \mid \otimes \zeta_{\iota}\right)=0$ and

$$
u \otimes \xi_{0 \iota}=\lambda \otimes \zeta_{\iota}+\zeta
$$

for $\lambda>0$. If $c \neq c^{\prime}$, we have an $\varepsilon$ in $(0,1)$ such that for any $J_{0} \subset \subset I$ there exists a $J_{1} \subset \subset I \backslash J_{0}$ satisfying $\left|\Pi_{J_{1}}\left(s\left(x_{\iota}\right) \xi_{0} \mid \zeta_{\iota}\right)\right|<\varepsilon$. Choose $\lambda_{0}>1$ such that $\lambda_{0}^{-1} \leqq \prod_{J}\left\|\xi_{0}\right\| \leqq \lambda_{0}$ for all $J$. Then there exists an $n \in \boldsymbol{N}$ with $\varepsilon^{n}<\lambda \lambda_{0}^{-1}$ and a $K \subset \subset I$ such that $\left|\Pi_{K}\left(s\left(x_{\iota}\right) \xi_{0} \mid \zeta_{\iota}\right)\right|<\varepsilon^{n}$. Since $\lambda=$ $\left(u_{J} \otimes \xi_{0 \iota} \mid \otimes \zeta_{\iota}\right)$, we have

$$
\left|\left(u(I \backslash K) \underset{I \backslash K}{\otimes} \xi_{0 \iota} \mid \underset{I \backslash K}{\otimes} \zeta_{\iota}\right)\right|=\lambda\left|\prod_{K}\left(s\left(x_{\iota}\right) \xi_{0 \iota} \mid \zeta_{\iota}\right)\right|^{-1}>\lambda_{0},
$$

which is impossible. Thus $c=c^{\prime}$.

For $\otimes \xi_{\iota} \in \odot\left(D\left(x_{\iota}\right), \xi_{0 \iota}\right), J_{2}=\left\{\iota \in I: \xi_{\iota} \neq \xi_{0 \iota}\right\}$ and $\varepsilon>0$, we can choose a $J_{3} \subset \subset I$ with $J_{2} \subset J_{3}$ such that

$$
\left\|\left(\odot\left(x_{\iota}, \xi_{0 \iota}\right)-x_{K}\right) \otimes \xi_{0 \iota}\right\|<\varepsilon
$$

for any $J_{3} \subset K \subset \subset I$, where $x_{K}=\left(\otimes_{K} x_{\iota}\right) \otimes\left(\otimes_{I \backslash K}^{c} 1_{\imath}\right)$. Since $\otimes_{K} x_{\iota}$ is selfadjoint and $\bigodot_{K} D\left(x_{\iota}\right)$ is its core by Lemma 2.1, we have $\eta_{K}^{ \pm} \in \bigodot_{K} D\left(x_{\iota}\right)$ such that

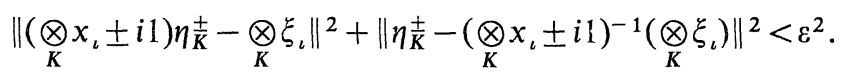

Put $\eta^{ \pm} \equiv \eta_{K}^{ \pm} \otimes\left(\otimes_{I \backslash K} \xi_{0_{\iota}}\right)$. From the above two inequalities we have

$$
\begin{aligned}
& \left\|\left(\odot\left(x_{\iota}, \xi_{0 \iota}\right) \pm i 1\right) \eta^{ \pm}-\otimes \xi_{\iota}\right\|^{-} \\
& \quad \leqq\left\|\left(\odot\left(x_{\imath}, \xi_{0 \iota}\right)-x_{K}\right) \eta^{ \pm}\right\|+\left\|\left(x_{K} \pm i 1\right) \eta^{ \pm}-\otimes \xi_{\iota}\right\|
\end{aligned}
$$




$$
\begin{aligned}
& \leqq\left\|\left(\otimes_{K}^{\otimes} x_{\iota}\right) \eta \bar{t}\right\|\left\|\left(\underset{I \backslash K}{\odot}\left(x_{\iota}, \xi_{0}\right)-1\right) \underset{I \backslash K}{\otimes} \xi_{0 \iota}\right\| \\
& +\left\|\underset{I \backslash K}{\otimes} \xi_{0 \iota}\right\|\left\|\left(\otimes_{K}^{\otimes} x_{\iota} \pm i 1\right) \eta \frac{ \pm}{K}-\underset{K}{\otimes} \xi_{\iota}\right\| \\
& \leqq \varepsilon\left\{2\left(\left\|\underset{K}{\otimes} \xi_{\iota}\right\|+\varepsilon\right)\left\|\underset{K}{\otimes} x_{\iota} \xi_{0 \iota}\right\|^{-1}+\left\|\underset{I \backslash K}{\otimes} \xi_{0 \iota}\right\|\right\} .
\end{aligned}
$$

Since there exists a $\lambda_{1}>1$ satisfying $\prod_{J}\left\|\xi_{0 \iota}\right\|<\lambda_{1}$ and $\lambda_{1}^{-1}<\prod_{J}\left\|x_{\iota} \xi_{0_{\iota}}\right\|$ for all $J \subset I$, we conclude that the deficiency indices of $\odot\left(x_{\iota}, \xi_{0}\right)$ are 0,0 and hence it is essentially self-adjoint. Furthermore $\odot\left(D\left(x_{\iota}\right), \xi_{0 \iota}\right)$ is a core of $x=\otimes^{c} x_{\iota}$.

Each $\check{\zeta}_{0}$ in $\odot\left(D\left(x_{\iota}\right), \xi_{0 \iota}\right)$ is of the form $\xi_{J} \otimes\left(\otimes_{I \backslash J} \xi_{0 \iota}\right)$ for some $J \subset \subset I$ and $\xi_{J} \in \otimes_{J} \mathfrak{Z} \mathcal{C}_{\iota}$. Since $\otimes_{J} x_{\iota}$ is positive, we have

$$
\left(x \xi_{0} \mid \xi_{0}\right)=\left(\left(\otimes_{J} x_{\iota}\right) \xi_{J} \mid \xi_{J}\right) \prod_{I}\left(x_{\iota} \xi_{0_{\iota}} \mid \xi_{0_{\iota}}\right) \geqq 0
$$

Since $\odot\left(D\left(x_{\iota}\right), \xi_{0 \iota}\right)$ is a core of $x, x$ is positive.

Q.E.D.

Remark 2.1. If $x_{\iota}$ is positive self-adjoint and if $\odot\left(x_{\iota}, \xi_{0_{\iota}}\right)$ is closable, then $\left(\xi_{0_{\iota}}, \xi_{0_{\iota}}\right)$ is a non-zero reference pair of $\left(x_{\iota}\right)$.

We may assume that $\left(x_{\iota} \xi_{0_{\iota}}\right) \in S_{0}$. If $\odot\left(x_{\iota}, \xi_{0_{\iota}}\right)$ is closable, then $\odot_{I \backslash J}\left(x_{\iota}, \xi_{0 \iota}\right)$ is closable for any $J \subset \subset I$ and hence

$$
\overline{\odot\left(\overline{x_{\iota}}, \xi_{0 \iota}\right)}=\left(\underset{J}{\otimes} x_{\iota}\right) \otimes\left(\overline{\left(\bigodot_{J}\left(x_{\iota}, \xi_{0 \iota}\right)\right.}\right) .
$$

Let

$$
\overline{\bigodot_{I \backslash J}\left(x_{\iota}, \xi_{0_{\iota}}\right)}=v(I \backslash J) x(I \backslash J)
$$

be the polar decomposition. It then follows from (2.1) that $v(I)=$ $\left(\otimes_{J} 1_{\iota}\right) \otimes v(I \backslash J)$. Since $\left(x_{\iota} \xi_{0 \iota}\right) \in S_{0}$, we may apply the similar argument as in the proof of Theorem 1.1 to these partial isometries and obtain that $\left(\xi_{0 \iota}\right) \sim\left(x_{\iota} \xi_{0 \iota}\right)$. Thus $\left(\xi_{0 \iota}, \xi_{0 \iota}\right)$ is a non-zero reference pair of $\left(x_{\iota}\right)$.

Example 2.2. For $\lambda>0$, put

$$
x_{\iota} \equiv\left(\begin{array}{cc}
\lambda & 0 \\
0 & \lambda^{-1}
\end{array}\right) \text { and } \xi_{\iota} \equiv\left(\begin{array}{c}
\left(1+\lambda^{2}\right)^{-1 / 2} \\
\lambda\left(1+\lambda^{2}\right)^{-1 / 2}
\end{array}\right) \text {. }
$$


Then $\left(\xi_{\iota}\right) \in S_{0}$ and $\left(x_{\iota} \xi_{\iota}\right) \in S_{0}$. Besides, if $\lambda \neq 1$, then $\left(x_{\iota}^{2} \xi_{\iota}\right) \notin S, \quad\left(\xi_{\iota}\right) x$ $\left(x_{\iota} \xi_{\iota}\right)$ and $\odot\left(x_{\iota}, \xi_{0 \iota}\right)$ is not closable.

Lemma 2.6. Let $\left(\xi_{0_{\iota}}, \eta_{0_{\iota}}\right)$ be a non-zero reference pair of $\left(x_{\iota}\right)$ and let $x_{\iota}=u_{\iota}\left|x_{\iota}\right|$ be the polar decomposition of $x_{\iota}$. Then

(i) $\left(u_{\iota}^{*} u_{\iota} \xi_{0_{\iota}}\right) \in S$ and $\left(u_{\iota} u_{\imath}^{*} \eta_{0 \iota}\right) \in S$;

(ii) $\left(\xi_{0 \iota}, \eta_{0 \iota}\right)$ and $\left(\eta_{0 \iota}, \xi_{0 \iota}\right)$ are non-zero reference pairs of $\left(u_{\iota}\right)$ and $\left(u_{\imath}^{*}\right)$, respectively;

(iii) $\left(\otimes{ }^{c^{\prime} c} u_{\iota}\right)^{*}=\otimes{ }^{c c^{\prime}} u_{\iota}^{*}$; and

(iv) if $\left(u_{\iota}^{*} u_{\iota} \xi_{0 \iota}\right) \in S_{0}$ and $\left(u_{\iota} u_{\iota}^{*} \eta_{0 \iota}\right) \in S_{0},\left(u_{\iota} \xi_{0 \iota}, u_{\imath}^{*} \eta_{0 \iota}\right)$ is a non-zero reference pair of $\left(x_{\iota}^{*}\right)$.

Proof. (i) Since $\left(x_{\iota}^{*} \eta_{0 \iota}\right) \in S$, we have $\left(\left|x_{\iota}^{*}\right| \eta_{0 \iota}\right) \in S$. Since $\left(x_{\iota}^{*} \eta_{0_{\iota}}\right) \sim$ $\left(\xi_{0_{\iota}}\right)$, we have $\left(\left|x_{\iota}^{*}\right| \eta_{0_{\iota}}\right) \sim\left(u_{\iota} \xi_{0_{\iota}}\right)$. Since $\left\|u_{\iota}\right\|=1$ and $\left(\xi_{0_{\iota}}\right) \in S$, it follows from Lemma 1 in [1] that $\left(u_{\iota} \xi_{0_{\iota}}\right) \in S$ and hence $\left(u_{\iota}^{*} u_{\iota} \xi_{0_{\iota}}\right) \in S$. Since $\left(x_{\iota} \xi_{0 \iota}\right) \in S$, we have $\left(u_{\iota} u_{\iota}^{*} \eta_{0}\right) \in S$.

(ii) $\left(u_{\iota} \xi_{0_{\iota}}\right) \in S$ and $\left(u_{\iota}^{*} \eta_{0_{\iota}}\right) \in S$ are shown in the above. Since $\left(\left|x_{\iota}^{*}\right| \eta_{0_{\iota}}\right) \sim\left(\eta_{0_{\iota}}\right)$ by Theorem 1.1, we have $\left(u_{\iota} \xi_{0_{\iota}}\right) \sim\left(\eta_{0_{\iota}}\right)$ and $\left(\xi_{0_{\iota}}\right) \sim\left(u_{\iota}^{*} \eta_{0_{\iota}}\right)$. Thus (ii) follows.

(iii) Sincc $\otimes c^{\prime} c u_{\iota}$ is bounded and since

$$
\begin{aligned}
& \left(\left(\otimes c^{\prime} c u_{\iota}\right) \otimes \xi_{\iota} \mid \otimes \eta_{\iota}\right)=\left(\otimes u_{\iota} \xi_{\iota} \mid \otimes \eta_{\iota}\right) \\
& =\prod\left(u_{\iota} \xi_{\iota} \mid \eta_{\iota}\right)=\prod\left(\xi_{\iota} \mid u_{\iota}^{*} \eta_{\iota}\right) \\
& =\left(\otimes \xi_{\iota} \mid \otimes u_{\iota}^{*} \eta_{\iota}\right)=\left(\otimes \xi_{\iota} \mid\left(\otimes c c^{\prime} u_{\iota}^{*}\right) \otimes \eta_{\iota}\right)
\end{aligned}
$$

for all $\otimes \xi_{\iota} \in \odot\left(D\left(x_{\iota}\right), \xi_{0 \iota}\right)$ and $\otimes \eta_{\iota} \in \odot\left(D\left(x_{\iota}^{*}\right), \eta_{0 \iota}\right)$, we have (iii).

(iv) Since $x_{\iota}^{*} u_{\iota} \xi_{0_{\iota}}=\left|x_{\iota}\right| \xi_{0 \iota}$ and $x_{\iota} u_{\iota}^{*} \eta_{0_{\iota}}=\left|x_{\iota}^{*}\right| \eta_{0_{\iota}},\left(u_{\iota} \xi_{0_{\iota}}, u_{\iota}^{*} \eta_{0_{\iota}}\right)$ is a non-zero reference pair of $\left(x_{\iota}^{*}\right)$.

Q.E.D.

Theorem 2.1. Let $\left(\xi_{0_{\iota}}, \eta_{0_{\iota}}\right)$ be a non-zero reference pair of $\left(x_{\iota}\right)$ and let $x_{\iota}=u_{\imath}\left|x_{\imath}\right|$ be the polar decomposition of $x_{\iota}$. Then

$$
\begin{aligned}
\otimes{ }^{c^{\prime} c} x_{\iota} & =\left(\otimes c^{c^{\prime} c} u_{\iota}\right)\left(\otimes{ }^{c}\left|x_{\iota}\right|\right) \\
& =\left(\otimes c^{\prime}\left|x_{\iota}^{*}\right|\right)\left(\otimes c^{c^{\prime}} u_{\iota}\right)
\end{aligned}
$$

and (2.2) is the polar decomposition of $\otimes c^{\prime} c x_{1}$, where $c \equiv c\left(\xi_{0,}\right)$ and 
$c^{\prime} \equiv c\left(\eta_{0_{\iota}}\right)$

Proof. Since $D\left(x_{\iota}\right)=D\left(\left|x_{\iota}\right|\right)$, we have $\odot\left(D\left(x_{\iota}\right), \xi_{0 \iota}\right)=\odot\left(D\left(\left|x_{\iota}\right|\right), \xi_{0 \iota}\right)$. From Theorem 1.1 we have $\left(\xi_{0_{\iota}}\right) \sim\left(\left|x_{\iota}\right| \xi_{0_{\iota}}\right)$. Since $\left(\xi_{0_{\iota}}, \eta_{0_{\iota}}\right)$ is a non-zero reference pair of $\left(u_{\iota}\right)$ by Lemma 2.6 and since $\left\|u_{\imath}\right\| \leqq 1$, we find that $\left(\left|x_{\iota}\right| \xi_{0 \iota}, \eta_{0}\right)$ is also a non-zero reference pair of $\left(u_{\iota}\right)$ and that $\otimes{ }^{c^{\prime}} c_{\iota}$ is the closure of $\odot\left(u_{\imath},\left|x_{\iota}\right| \xi_{0_{\iota}}\right)$. We have

$$
\begin{aligned}
& \left(\otimes c^{\prime} c x_{\imath}\right) \otimes \xi_{\iota}=\otimes x_{\iota} \xi_{\iota}=\otimes u_{\imath}\left|x_{\imath}\right| \xi_{\iota} \\
& =\left(\otimes c^{\prime} c_{\imath}\right) \otimes\left|x_{\iota}\right| \xi_{\iota}=\left(\otimes^{c^{\prime}} c_{\imath}\right)\left(\otimes c\left|x_{\iota}\right|\right) \otimes \xi_{\iota}
\end{aligned}
$$

for all $\otimes \xi_{\iota} \in \odot\left(D\left(x_{\iota}\right), \xi_{0 \iota}\right)$. Since $\odot\left(D\left(x_{\iota}\right), \xi_{0 \iota}\right)$ is a core of $\otimes^{c^{\prime} c} x_{\iota}$ and $\otimes^{c}\left|x_{\iota}\right|$, we have (2.2).

Since $x_{\iota}=u_{\iota}\left|x_{\iota}\right|$ is a polar decomposition of $x_{\iota}, u_{\iota}^{*} u_{\iota}$ is a projection onto the closure of the range of $\left|x_{\iota}\right|$. Since $\left(\xi_{0 \iota}\right) \sim\left(\left|x_{\iota}\right| \xi_{0_{\iota}}\right), \otimes{ }^{c} u_{\iota}^{*} u_{\iota} \mathscr{H}_{\iota}$ is the closed linear span of

$$
\left\{\otimes\left|x_{\iota}\right| \xi_{\iota}: \otimes \xi_{\iota} \in \odot\left(x_{\iota}, \xi_{0 \iota}\right)\right\}
$$

Therefore the closure of the range of $\otimes^{c}\left|x_{\iota}\right|$ is the initial space of a partial isometry $\otimes c^{\prime} c u_{\iota}$. Thus (2.2) is the polar decomposition.

(2.3) is proved similarly. Since $\left(x_{\iota} \xi_{0 \iota}\right) \in S$ and $\left(x_{\iota}^{*} \eta_{0_{\iota}}\right) \in S$, we may assume that $\otimes x_{\iota} \xi_{0 \iota} \neq 0$ and $\otimes x_{\iota}^{*} \eta_{0 \iota} \neq 0$ by the same reason at the beginning part of the proof of Theorem 1.1. Therefore $\left(u_{\iota}^{*} u_{\iota} \xi_{0_{\iota}}\right) \in S_{0}$ and $\left(u_{\iota} u_{\imath}^{*} \eta_{0_{\iota}}\right) \in S_{0}$ as above. From Lemma 2.6 it follows that $\left(u_{\iota} \xi_{0_{\iota}}, u_{\iota}^{*} \eta_{0_{\iota}}\right)$ is a non-zero reference pair of $\left(x_{\iota}^{*}\right)$ and hence from Theorem 1.1 that $\left(u_{\iota} \xi_{0 \iota}, u_{\iota} \xi_{0}\right)$ is a non-zero reference pair of $\left(\left|x_{\iota}^{*}\right|\right)$. Since $\left|x_{\iota}\right|=u_{\iota}^{*}\left|x_{\iota}^{*}\right| u_{\iota}$, we have $u_{\imath} D\left(x_{\iota}\right)=u_{\iota} D\left(\left|x_{\iota}\right|\right)=D\left(\left|x_{\imath}^{*}\right|\right)$. This implies $\left(\otimes c^{\prime} c_{\iota}\right) \odot\left(D\left(x_{\iota}\right), \xi_{0_{\iota}}\right)=$ $\odot\left(D\left(\left|x_{\iota}^{*}\right|\right), u_{\iota} \xi_{0}\right)$. Hence we have

$$
\begin{aligned}
\left(\otimes c^{\prime} c x_{\iota}\right) \otimes \xi_{\iota} & =\otimes\left|x_{\iota}^{*}\right| u_{\iota} \xi_{\iota}=\left(\otimes c^{\prime}\left|x_{\iota}^{*}\right|\right) \otimes u_{\iota} \xi_{\iota} \\
& =\left(\otimes c^{\prime}\left|x_{\iota}^{*}\right|\right)\left(\otimes c^{c^{\prime}} u_{\iota}\right) \otimes \xi_{\iota}
\end{aligned}
$$

for all $\otimes \xi_{\iota} \in \odot\left(D\left(x_{\iota}\right), \xi_{0 \iota}\right)$. Since $\odot\left(D\left(x_{\iota}\right), \xi_{0 \iota}\right)$ is a core of $\otimes c^{c^{\prime}} x_{\iota}$ and $\left(\otimes c^{c^{\prime}} u_{\iota}\right) \odot\left(D\left(x_{\iota}\right), \xi_{0}\right)$ is a core of $\otimes^{c^{\prime}}\left|x_{\iota}^{*}\right|$, we have (2.3).

Q.E.D. 
Remark 2.2. If $\odot\left(x_{\iota}, \breve{\zeta}_{0_{\iota}}\right)$ is closable, then there exists a $C_{0}$-sequence $\left(\eta_{0}\right) \in S_{0}$ such that $\left(\xi_{0_{\iota}}, \eta_{0_{\iota}}\right)$ is a non-zero reference pair fo $\left(x_{\iota}\right)$. This is proved by combining Remark 1.1 and Theorem 2.1.

Theorem 2.2. Under the same assumption as Theorem 2.1,

$$
\left(\otimes c^{\prime} c x_{\iota}\right)^{*}=\otimes c c^{\prime} x_{\iota}^{*}
$$

Proof. Using (2.3) and (iii) of Lemma 2.5, we have

$$
\left(\otimes c^{\prime} c x_{\iota}\right)^{*}=\left(\otimes c^{c^{\prime} c} u_{\iota}\right)^{*}\left(\otimes c^{\prime}\left|x_{\iota}^{*}\right|\right)=\left(\otimes c c^{\prime} u_{\iota}^{*}\right)\left(\otimes c^{\prime}\left|x_{\iota}^{*}\right|\right) \text {. }
$$

Since $x_{\iota}^{*}=u_{\imath}^{*}\left|x_{\iota}^{*}\right|$ and $\left(\eta_{0 \iota}, \xi_{0 \iota}\right)$ is a non-zero reference pair of $\left(x_{\iota}^{*}\right)$ by Lemma 2.3, we have $\otimes c c^{\prime} x_{\iota}^{*}=\left(\otimes c c^{\prime} u_{\iota}^{*}\right)\left(\otimes c^{\prime}\left|x_{\imath}^{*}\right|\right)$ by (2.2). This completes the proof.

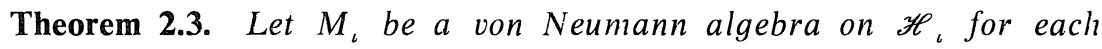
$\iota \in I$, and let $x_{\iota}$ be an operator affiliated with $M_{\iota}$. If $\left(\xi_{0_{\iota}}, \eta_{0 \iota}\right)$ is a non-zero reference pair of $\left(x_{\iota}\right)$ with $c\left(\xi_{0_{\iota}}\right)=c\left(\eta_{0_{\iota}}\right)=c$, then $\otimes^{c} x_{\iota}$ is affiliated with $\otimes^{c} M_{\imath}$.

Proof. If $\xi \in D\left(\otimes^{c} x_{\iota}\right)$, there exists a sequence $\left\{\xi_{n}\right\}_{n=1}^{\infty}$ in $\odot\left(D\left(x_{\iota}\right)\right.$, $\left.\xi_{0 \iota}\right)$ such that $\xi_{n} \rightarrow \xi$ and $\left(\otimes^{c} x_{\iota}\right) \xi_{n} \rightarrow\left(\otimes{ }^{c} x_{\iota}\right) \xi$ in $\mathscr{H}_{c}$. According to Lemma 6.10 in [2], we have $\left(\otimes^{c} M_{\iota}\right)^{\prime}=\otimes^{c} M_{\iota}^{\prime}$ and hence $\otimes^{c} M_{\iota}^{\prime}$ is generated by $\otimes{ }^{c} v_{\iota}$ such that $v_{\iota}$ is a unitary in $M_{\iota}^{\prime}$ and $v_{\iota}=1$ except for a finite number of e. For each $\xi_{n}$ of the form $\xi_{n}=\sum_{j=1}^{m} \otimes \xi_{j_{\iota}}$ with $\otimes \xi_{j \iota} \in$ $\odot\left(D\left(x_{\iota}\right), \xi_{0 \iota}\right)$, we find $\left(\otimes{ }^{c} v_{\iota}\right) \xi_{n}=\sum_{j=1}^{m} \otimes v_{\iota} \xi_{j \iota}$ in $\odot\left(D\left(x_{\iota}\right), \xi_{0_{\iota}}\right)$. This shows that $\odot\left(D\left(x_{\iota}\right), \xi_{0 \iota}\right)$ is invariant under such $\otimes v_{\iota}$ and hence $D\left(\otimes \otimes^{c} x_{\iota}\right)$ is invariant under $\otimes^{c} M_{\iota}^{\prime}$. It follows that $\left\{\left(\otimes^{c} v_{\iota}\right) \xi_{n}\right\}_{n=1}$ is a Cauchy sequence in $\odot\left(D\left(x_{\iota}\right), \xi_{0 \iota}\right)$ in the sense of graph of $\otimes x^{c} x_{\iota}$. Thus

$$
\begin{aligned}
& \left(\otimes^{c} x_{\iota}\right)\left(\otimes^{c} v_{\iota}\right) \xi=\lim _{n \rightarrow \infty}\left(\otimes^{c} x_{\imath}\right)\left(\otimes^{c} v_{\iota}\right) \xi_{n} \\
& =\lim _{n \rightarrow \infty}\left(\otimes^{c} v_{\iota}\right)\left(\otimes^{c} x_{\iota}\right) \xi_{n}=\left(\otimes^{c} v_{\iota}\right)\left(\otimes^{c} x_{\iota}\right) \xi,
\end{aligned}
$$

which shows that $\otimes^{c} x_{n}$ is affiliated with $\otimes^{c} M_{\prime \prime}$.

Q.E.D. 


\section{§3. Conditions for the Existence of Reference Pair}

We shall give some conditions for the existence of a non-zero reference pair of invertible, positive and self-adjoint operators $\left(x_{\iota}\right)$ in the following theorem. With a slight modification on convergence, the condition (iv) is known as Kolmogorov's three series theorem and the condition (vi) is interpreted as follows: the product of characteristic functions is also a characteristic function.

Theorem 3.1. Let $x_{\iota}$ be an invertible, positive and self-adjoint operator on $\mathscr{H}_{\iota}$ for $\iota \in I$ and $y_{\iota} \equiv \log x_{\iota}$. Let $e_{\iota}$ be the spectral projection of $x_{\iota}$ corresponding to the interval $\left[\lambda_{0}^{-1}, \lambda_{0}\right]$ for any fixed $\lambda_{0}>1$. The following six conditions are equivalent for $c \in C$ :

(i) there exists a non-zero reference pair $\left(\xi_{0_{\iota}}, \xi_{0_{\iota}}\right)$ of $\left(x_{\iota}\right)$ with $c=c\left(\xi_{0}\right)$;

(ii) $\left(e_{\iota} \xi_{1}\right) \in S, \quad\left(x_{\iota} e_{\iota} \xi_{1 \iota}\right) \in S$ and $\left(e_{\iota} \xi_{1 \iota}\right) \sim\left(x_{\iota} e_{\iota} \xi_{1 \iota}\right)$ hold for some $\left(\xi_{1}\right) \in c$;

(iii) $\left(e_{\iota} \xi_{\iota}\right) \in S, \quad\left(x_{\iota} e_{\iota} \xi_{\iota}\right) \in S$ and $\left(e_{\iota} \xi_{\iota}\right) \sim\left(x_{\iota} e_{\iota} \xi_{\iota}\right)$ hold for all $\left(\xi_{\iota}\right) \in c$;

(iv) $\left(e_{\iota} \xi_{\iota}\right) \in S, \sum\left\|y_{\iota} e_{\iota} \xi_{\iota}\right\|^{2}<+\infty$ and $\sum\left|\left(y_{\iota} e_{\iota} \xi_{\iota} \mid \xi_{\iota}\right)\right|<+\infty$ hold for all $\left(\xi_{\iota}\right) \in c$;

(v) $\xi_{2 \iota} \in D\left(y_{\imath}\right), \quad \sum\left\|y_{\imath} \xi_{2 \iota}\right\|^{2}<+\infty$ and $\sum\left|\left(y_{\imath} \xi_{2_{\imath}} \mid \xi_{2_{\imath}}\right)\right|<+\infty$ hold for some $\left(\xi_{2 \iota}\right) \in c$; and

(vi) $\otimes^{c} x_{t}^{i t}, t \in \mathbb{R}$ is a strongly continuous one parameter unitary group.

Proof. (i) $\Rightarrow$ (ii). We put $\xi_{1 \iota} \equiv \xi_{0 \iota}$ for all $\iota$. Since $\left(\check{\zeta}_{0_{\iota}}, \breve{\zeta}_{0_{\iota}}\right)$ is a non-zero reference pair of $\left(x_{\iota}\right)$, we have

$$
\sum\left|\left\|x_{\iota} \xi_{1 \iota}\right\|^{2}-1\right|<+\infty \text { and } \sum\left|\left(x_{\iota} \xi_{1} \mid \xi_{1 \iota}\right)-1\right|<+\infty \text {, }
$$

which imply

$$
\sum\left\|\left(1-x_{\iota}\right) \xi_{1 \iota}\right\|^{2}<+\infty \quad \text { and } \quad \sum\left|\left(\left(1-x_{\iota}\right) \xi_{1}, \xi_{1}\right)\right|<+\infty \text {. }
$$

Since $\left(1-\lambda_{0}^{-1}\right)\left(1-e_{\iota}\right) \leqq\left|1-x_{\iota}\right|\left(1-e_{\iota}\right)$, we have

$$
\left(\left(1-e_{\iota}\right) \xi_{1_{\iota}} \mid \xi_{1_{\iota}}\right) \leqq\left(1-\lambda_{0}^{-1}\right)^{-2}\left(\left(1-x_{\iota}\right)^{2}\left(1-e_{,}\right) \check{\zeta}_{1_{\iota}} \mid \xi_{1^{\prime}}\right)
$$


and

$$
\begin{aligned}
& \left|\left(\left(1-x_{\iota}\right) e_{\iota} \xi_{1 \iota} \mid \xi_{1 \iota}\right)\right| \\
& \quad \leqq\left|\left(\left(1-x_{\iota}\right) \xi_{1 \iota} \mid \xi_{1 \iota}\right)\right|+\left(1-\lambda_{0}^{-1}\right)^{-1}\left(\left(1-x_{\iota}\right)^{2}\left(1-e_{\iota}\right) \xi_{1_{\iota}} \mid \xi_{1 \iota}\right) .
\end{aligned}
$$

Since $\left\|\left(1-x_{\iota}\right) e_{\iota} \xi_{1 \iota}\right\| \leqq\left\|\left(1-x_{\iota}\right) \dot{\xi}_{1 \iota}\right\|$ and $\left\|\left(1-x_{\iota}\right)\left(1-e_{\iota}\right) \xi_{1 \iota}\right\| \leqq\left\|\left(1-x_{\iota}\right) \xi_{1 \iota}\right\|$, it follows from

$$
\left|\left\|e_{\iota} \xi_{1 \iota}\right\|^{2}-1\right| \leqq\left\|\left(1-e_{\iota}\right) \xi_{1 \iota}\right\|^{2}+\left|\left\|\xi_{1 \iota}\right\|^{2}-1\right|
$$

and

$$
\begin{aligned}
& \left|\left\|x_{\imath} e_{\iota} \xi_{1 \iota}\right\|^{2}-1\right| \\
& \quad \leqq\left|\left\|e_{\iota} \xi_{1 \iota}\right\|^{2}-1\right|+2\left|\left(\left(1-x_{\iota}\right) e_{\iota} \check{\zeta}_{1} \iota \xi_{1 \iota}\right)\right|+\left\|\left(1-x_{\iota}\right) e_{\iota} \xi_{1 \iota}\right\|^{2}
\end{aligned}
$$

that $\left(e_{\imath} \xi_{1 \iota}\right) \in S,\left(x_{\imath} e_{\imath} \xi_{1 \iota}\right) \in S$ and $\left(e_{\iota} \breve{\zeta}_{1 \iota}\right) \sim\left(x_{\imath} e_{\iota} \xi_{1 \iota}\right)$.

(ii) $\Rightarrow$ (iii). $\quad\left(e_{\iota} \xi_{1 \iota}\right) \in S$ implies $\left(\xi_{1 \iota}\right) \sim\left(e_{\iota} \xi_{1 \iota}\right)$. If $\left(\xi_{\iota}\right) \in c$, then $\left(\xi_{\iota}\right) \sim$ $\left(\xi_{1}\right)$. Therefore $\left(e_{\iota} \xi_{\iota}\right) \in S$ by Lemma 1 in [1]. Since $\left(\xi_{\iota}\right) \sim\left(\xi_{1 \iota}\right)$, we have $\sum\left\|\xi_{\iota}-\xi_{1}\right\|^{2}<+\infty$. Since

$$
\begin{aligned}
\left\|\left(1-x_{\iota}\right) e_{\iota} \xi_{\iota}\right\|^{2} & \leqq 2\left(\left\|\left(1-x_{\iota}\right) e_{\iota} \xi_{1 \iota}\right\|^{2}+\left\|\left(1-x_{\iota}\right) e_{\iota}\left(\xi_{\iota}-\xi_{1 \iota}\right)\right\|^{2}\right) \\
& \leqq 2\left(\left\|\left(1-x_{\iota}\right) e_{\iota} \xi_{1}\right\|^{2}+\left(\lambda_{0}-1\right)\left\|\xi_{\iota}-\xi_{1 \iota}\right\|^{2}\right),
\end{aligned}
$$

we have $\sum\left\|\left(1-x_{\iota}\right) c_{\iota} \xi_{\iota}\right\|^{2}<+\infty$. Sincc

$$
\begin{aligned}
& \left|\left(\left(1-x_{\iota}\right) e_{\iota} \xi_{\iota} \mid \xi_{\iota}\right)\right| \\
& \quad \leqq\left|\left(\left(1-x_{\iota}\right) e_{\iota} \xi_{1 \iota} \mid \xi_{1 \iota}\right)\right|+\left(\left\|\left(1-x_{\iota}\right) e_{\iota} \xi_{\iota}\right\|+\left\|\left(1-x_{\iota}\right) e_{\iota} \xi_{1 \iota}\right\|\right)\left\|\xi_{\iota}-\xi_{1 \iota}\right\| \\
& \quad \leqq\left|\left(\left(1-x_{\iota}\right) e_{\iota} \xi_{1} \mid \xi_{1}\right)\right|+\left\|\left(1-x_{\iota}\right) e_{\iota} \xi_{\iota}\right\|^{2}+\left\|\left(1-x_{\iota}\right) e_{\iota} \xi_{1}\right\|\left\|^{2}+\right\| \xi_{\iota}-\xi_{1} \|^{2},
\end{aligned}
$$

we have $\sum\left|\left(\left(1-x_{\imath}\right) e_{\iota} \xi_{\iota} \mid \xi_{\iota}\right)\right|<+\infty$. Consequently, $\left(x_{\iota} e_{\iota} \xi_{\iota}\right) \in S$ and $\left(e_{\iota} \xi_{\iota}\right) \sim$ $\left(x_{\iota} e_{\iota} \xi_{\iota}\right)$.

(iii) $\Rightarrow$ (i). Since $\left(e_{\iota} \xi_{\iota}\right) \in S$, we may assume that $\left(e_{\iota} \xi_{\iota}\right) \in S_{0}$. Set $\xi_{0 \iota} \equiv$ $e_{\iota} \xi_{\iota}$. It then follows that $\left(\xi_{0 \iota}\right) \in c$ and that $\left(\xi_{0_{\iota}}, \xi_{0_{\iota}}\right)$ is a non-zero reference pair of $\left(x_{\iota}\right)$.

(iii) $\Rightarrow$ (iv). Since

$$
-x_{,}^{-1}\left(1-x_{1}\right)=-\left(x_{,}^{-1}-1\right) \leqq y_{1} \leqq x_{1}-1
$$


and

$$
\left|y_{\iota}-\left(x_{\iota}-1\right)\right| e_{\iota} \leqq \lambda_{1}\left(x_{\iota}-1\right)^{2} e_{\iota}
$$

for some constant $\lambda_{1}>0$, we have

$$
\left|\left(y_{\iota} e_{\iota} \xi_{\iota} \mid \xi_{\iota}\right)\right| \leqq\left|\left(\left(x_{\iota}-1\right) e_{\iota} \xi_{\iota} \mid \xi_{\iota}\right)\right|+\lambda_{1}\left\|\left(x_{\iota}-1\right) e_{\iota} \xi_{\iota}\right\|^{2}
$$

and

$$
\begin{aligned}
\left\|y_{\iota} e_{\iota} \xi_{\iota}\right\|^{2} & \leqq\left\|\left(x_{\iota}-1\right) e_{\iota} \xi_{\iota}\right\|^{2}+\left\|x_{\iota}^{-1}\left(1-x_{\iota}\right) e_{\iota} \xi_{\iota}\right\|^{2} \\
& \leqq\left(1+\lambda_{0}^{2}\right)\left\|\left(1-x_{\iota}\right) e_{\iota} \xi_{\iota}\right\|^{2} .
\end{aligned}
$$

Since $\left(e_{\iota} \xi_{\iota}\right) \in S,\left(x_{\iota} e_{\iota} \xi_{\iota}\right) \in S$ and $\left(\xi_{\iota}\right) \sim\left(x_{\iota} e_{\iota} \xi_{\iota}\right)$ from (iii), the right hand sides of these inequalities are summable over $\iota \in I$. Thus (iv) follows. (iv) $\Rightarrow$ (iii). Since $-x_{\iota} y_{\iota} \leqq 1-x_{\iota} \leqq-y_{\iota}$ and $\left|1-x_{\iota}-\left(-y_{\iota}\right)\right| e_{\iota} \leqq \lambda_{2} y_{\iota}^{2} e_{\iota}$ for some constant $\lambda_{2}>0$, we have

$$
\left|\left(\left(1-x_{\iota}\right) e_{\iota} \xi_{\iota} \mid \xi_{\iota}\right)\right| \leqq\left|\left(y_{\iota} e_{\iota} \xi_{\iota} \mid \xi_{\iota}\right)\right|+\lambda_{2}\left\|y_{\iota} e_{\iota} \xi_{\iota}\right\|^{2}
$$

and

$$
\left\|\left(1-x_{\iota}\right) e_{\iota} \xi_{\iota}\right\|^{2} \leqq\left\|y_{\iota} e_{\iota} \xi_{\iota}\right\|^{2}+\left\|x_{\iota} y_{\iota} e_{\iota} \xi_{\iota}\right\|^{2} \leqq\left(1+\lambda_{0}^{2}\right)\left\|y_{\iota} e_{\iota} \xi_{\iota}\right\|^{2}
$$

Thus we have (iii) from (iv).

(iv) $\Rightarrow$ (v). Putting $\xi_{2 \iota}=e_{\iota} \xi_{\iota}$, we have (v) from (iv).

(v) $\Rightarrow$ (vi). If $\left(\xi_{\iota}\right) \in c$, then $\left(\xi_{\iota}\right) \sim\left(\xi_{2_{\iota}}\right)$. Since $\log \lambda_{0}\left(1-e_{\iota}\right) \leqq\left|y_{\iota}\right|\left(1-e_{\iota}\right)$, we have

$$
\left(\left(1-e_{\iota}\right) \xi_{2 \iota} \mid \xi_{2 \iota}\right) \leqq\left(\log \lambda_{0}\right)^{-2}\left\|y_{\iota}\left(1-e_{\iota}\right) \xi_{2 \iota}\right\|^{2}
$$

Since $\left\|y_{\iota}\left(1-e_{\iota}\right) \xi_{2_{\imath}}\right\| \leqq\left\|y_{\imath} \xi_{2_{\imath}}\right\|$, we have $\left(e_{\imath} \xi_{2_{\imath}}\right) \in S$. Since $\left(\xi_{\iota}\right) \sim\left(e_{\imath} \xi_{2 \iota}\right)$, $\left(e_{\iota} \xi_{\iota}\right) \in S$. Since $\left(\xi_{\iota}\right) \sim\left(\xi_{2 \iota}\right), \quad \sum\left\|\xi_{\iota}-\xi_{2 \iota}\right\|^{2}<+\infty$. Since

$$
\begin{aligned}
\left\|y_{\iota} e_{\iota} \xi_{\iota}\right\|^{2} & \leqq 2\left(\left\|y_{\iota} e_{\iota} \xi_{2 \iota}\right\|^{2}+\left\|y_{\iota} e_{\iota}\left(\xi_{\iota}-\xi_{2 \iota}\right)\right\|^{2}\right) \\
& \leqq 2\left(\left\|y_{\iota} \xi_{2_{\iota}}\right\|^{2}+\left(\log \lambda_{0}\right)^{2}\left\|\xi_{\iota}-\xi_{2 \iota}\right\|\right)^{2},
\end{aligned}
$$

we have $\sum\left\|y_{\imath} e_{\imath} \xi_{\imath}\right\|^{2}<+\infty$. Since 


$$
\begin{aligned}
& \left|\left(y_{\iota} e_{\iota} \xi_{\iota} \mid \xi_{\iota}\right)\right| \\
& \quad \leqq\left|\left(y_{\iota} e_{\iota} \xi_{2_{\iota}} \mid \xi_{2 \iota}\right)\right|+\left(\left\|y_{\iota} e_{\iota} \xi_{\iota}\right\|+\left\|y_{\iota} e_{\iota} \xi_{2_{\iota}}\right\|\right)\left\|\xi_{\iota}-\xi_{2 \iota}\right\| \\
& \quad \leqq\left|\left(y_{\iota} e_{\iota} \xi_{2_{\iota}} \mid \xi_{2 \iota}\right)\right|+\left\|y_{\iota} e_{\iota} \xi_{\iota}\right\|^{2}+\left\|y_{\iota} e_{\iota} \xi_{2 \iota}\right\|^{2}+\left\|\xi_{\iota}-\xi_{2_{\iota}}\right\|^{2},
\end{aligned}
$$

we have $\sum\left|\left(y_{\imath} e_{\imath} \xi_{\iota} \mid \xi_{\iota}\right)\right|<+\infty$.

(i) $\Rightarrow$ (iv). There is a countable subset $I_{0}$ of $I$ such that $\left\|\xi_{0}\right\|=1$, $\left\|x_{\iota} \xi_{0 \iota}\right\|=1$ and $\left(x_{\iota} \xi_{0 \iota} \mid \xi_{0 \iota}\right)=1$ for all $\iota \in I \backslash I_{0}$. Therefore $\left\|x_{\iota} \xi_{0 \iota}-\xi_{0_{\iota}}\right\|^{2}=0$ and hence $x_{\iota} \xi_{0 \iota}=\xi_{0 \iota}$ for all $\iota \in I \backslash I_{0}$. Therefore $x_{\iota}^{i t} \xi_{0 \iota}=\xi_{0 \iota}$ for $\iota \in I \backslash I_{0}$. Restricting the index set to $I_{0}$, we know that $\left(\xi_{0_{\iota}}, \xi_{0_{\iota}}\right)$ is a non-zero reference pair of $\left(x_{\iota} ; \iota \in I_{0}\right)$. Then $\otimes_{\left.I_{0}^{\left(\xi_{0}\right.}\right)}^{\left(y_{\imath}\right)} x_{\iota}^{i t}, t \in \boldsymbol{R}$ is strongly continuous by [14] and [13]. Since $\odot\left(\mathscr{H}_{\iota}, \xi_{0 \iota}\right)$ is dense in $\mathscr{H}_{c}$ and since $\otimes^{c} x_{\imath}^{i t}$ is bounded, it is strongly continuous unitary group in $t \in \mathbb{R}$.

(vi) $\Rightarrow(\mathrm{i})$. Choose $t_{0}$ and $t_{1}$ in $\boldsymbol{R}$ such that $t_{0} / t_{1}$ is irrational. For any $\left(\xi_{\iota}\right) \in S_{0}$ with $c=c\left(\xi_{\iota}\right)$, there exists a countable subset $I_{1}$ of $I$ such that $x_{\iota}^{i t_{0}} \xi_{\iota}=\xi_{\iota}$ and $x_{\iota}^{i t_{1}} \xi_{\iota}=\xi_{\iota}$ for all $\iota \in I \backslash I_{1}$. Then $x_{\iota}^{i t} \xi_{\iota}=\xi_{\iota}$ for all $t \in t_{0} \boldsymbol{Z}+t_{1} \boldsymbol{Z}$ and $\iota \in I \backslash I_{1}$. Since $t_{0} \boldsymbol{Z}+t_{1} \boldsymbol{Z}$ is dense in $\mathbb{R}$ and since $x_{\iota}^{i t}$ is strongly continuous in $t \in \boldsymbol{R}$, we find that $x_{\iota}^{i t} \xi_{\iota}=\xi_{\iota}$ for all $t \in \boldsymbol{R}$ and $\iota \in I \backslash I_{1}$. Applying [14] and [13] for this countable $I_{1}$, we have (iv) and hence (i) for $I_{1}$. Therefore there exists a non-zero reference pair $\left(\xi_{\iota}^{\prime}, \xi_{\iota}^{\prime}\right)$ of $\left(x_{\iota}\right)$ for $I_{1}$ and $\left(\xi_{\iota}^{\prime}\right) \sim\left(\xi_{\iota}\right)$ for $I_{1}$. Define $\left(\xi_{0_{\iota}}\right) \in S_{0}$ for $I$ by $\xi_{0 \iota} \equiv \xi_{\iota}$ for $\iota \in I \backslash I_{1}$ and $\xi_{0 \iota} \equiv \xi_{\iota}$ for $\iota \in I_{1}$. Then $\left(\xi_{0 \iota}\right) \in S_{0}, c=c\left(\xi_{0 \iota}\right)$, $\left(x_{\iota} \xi_{0 \iota}\right) \in S$ and $\left(\xi_{0 \iota}\right) \sim\left(x_{\iota} \xi_{0 \iota}\right)$. Consequently, $\left(\xi_{0 \iota}, \xi_{0 \iota}\right)$ is a non-zero reference pair of $\left(x_{\iota}\right)$ with $c=c\left(\xi_{0_{\iota}}\right)$.

Q.E.D.

Remark 3.1. Let $x_{\iota}$ be invertible, positive and self-adjoint, and $y_{\iota} \equiv \log x_{\iota}$. If $\left(\xi_{0_{\iota}}, \xi_{0_{\iota}}\right)$ is a non-zero reference pair of $\left(x_{\iota}\right)$, there exists a strong convergence vector $\otimes \xi_{2 \iota}$ of $\left(y_{\iota}\right)$ with $\left(\xi_{0 \iota}\right) \sim\left(\xi_{2 \iota}\right)$ in the sense of Reed, [13].

Theorem 3.2. If $\left(\xi_{0_{\iota}}, \eta_{0_{\iota}}\right)$ is a non-zero reference pair of $\left(x_{\iota}\right)$, there exists a non-zero reference pair $\left(\xi_{1 \iota}, \xi_{1 \iota}\right)$ of $\left(x_{\imath}^{*} x_{\iota}\right)$ with $\left(\xi_{1}\right) \sim$ $\left(\xi_{0 \iota}\right)$ and

$$
\otimes{ }^{c} x_{\iota}^{*} x_{\iota}=\left(\otimes c^{\prime} c x_{\iota}\right)^{*}\left(\otimes c^{\prime} c x_{\iota}\right)
$$

Proof. If $\left(\xi_{0 \iota}, \eta_{0 \iota}\right)$ is a non-zero reference pair of $\left(x_{\iota}\right)$, then $\left(\xi_{0_{\iota}}\right.$, 
$\left.\xi_{0_{\iota}}\right)$ is a non-zero reference pair of $\left(\left|x_{\iota}\right|\right)$. Since $\left(\operatorname{Ker} \otimes^{c} x_{\iota}^{*} x_{\iota}\right)^{\perp} \subset(\operatorname{Ker}$ $\left.\otimes^{c^{\prime} c} x_{\iota}\right)^{\perp}=\otimes^{c}\left(\operatorname{Ker} x_{\iota}\right)^{\perp}$, we can restrict our proof over $\otimes^{c}\left(\operatorname{Ker} x_{\iota}\right)^{\perp}$. By the implication (i) $\Rightarrow$ (vi) of Theorem 3.1, $\otimes^{c}\left|x_{\iota}\right|^{i t}$ is strongly continuous unitary group in $t \in \boldsymbol{R}$ for $c=c\left(\xi_{0_{\iota}}\right)$. Since $\otimes^{c}\left(x_{\iota}^{*} x_{\iota}\right)^{i t}=\otimes^{c}\left|x_{\iota}\right|^{2 i t}$, by the implication (vi) $\Rightarrow$ (i) of Theorem 3.1 we have a non-zero reference pair $\left(\xi_{1 \iota}, \xi_{1 \imath}\right)$ of $\left(x_{\imath}^{*} x_{\iota}\right)$ with $c=c\left(\xi_{1 \imath}\right)$. Since $\left(x_{\imath}^{*} x_{\iota} \xi_{1 \iota}\right) \in S$, we may assume that $\left(x_{\imath}^{*} x_{\iota} \xi_{1 \iota}\right) \in S_{0}$. Since $D\left(x_{\iota}^{*} x_{\iota}\right) \subset D\left(\left|x_{\iota}\right|\right)$ and since

$$
\begin{aligned}
& \left\|\left(\left|x_{\iota}\right|-1\right) \xi_{1 \iota}\right\|^{2} \leqq\left\|\left(x_{\iota}^{*} x_{\iota}-1\right) \xi_{1 \iota}\right\|^{2} \\
& =\left\|x_{\iota}^{*} x_{\iota} \xi_{1 \iota}\right\|^{2}-2\left\|x_{\iota} \xi_{1 \iota}\right\|^{2}+\left\|\xi_{1 \iota}\right\|^{2},
\end{aligned}
$$

we have $\left(\xi_{1 \iota}\right) \sim\left(\left|x_{\iota}\right| \xi_{1 \iota}\right)$. Therefore $\left(\xi_{1 \iota}, \xi_{1 \iota}\right)$ is also a non-zero reference pair of $\left(\left|x_{\iota}\right|\right)$. Since $\left(x_{\iota}^{*} x_{\iota} \xi_{1 \iota}\right) \in S_{0}$ and $\left(x_{\iota} \xi_{1 \iota}\right) \in S_{0}$, we find that $\left(x_{\iota} \xi_{1 \iota}\right.$, $\left.\xi_{1 \iota}\right)$ is a non zero-reference pair of $\left(x_{\iota}^{*}\right)$ and

$$
\left(\otimes c^{\prime} c x_{\iota}\right) \odot\left(D\left(x_{\iota}^{*} x_{\iota}\right), \xi_{1 \iota}\right) \subset \odot\left(D\left(x_{\iota}^{*}\right), x_{\iota} \xi_{1 \iota}\right) .
$$

Therefore $\odot\left(D\left(x_{\imath}^{*} x_{\iota}\right), \xi_{1}\right)$ is included in the domain of $\left(\otimes c^{\prime} c x_{\iota}\right)^{*}\left(\otimes c^{\prime} x_{\iota}\right)$. Since (3.1) holds on $\odot\left(D\left(x_{\imath}^{*} x_{\iota}\right), \xi_{1}\right)$, we have

$$
\otimes{ }^{c} x_{\iota}^{*} x_{\iota} \subset\left(\otimes c^{\prime} c x_{\iota}\right)^{*}\left(\otimes c^{\prime} c x_{\iota}\right) \text {. }
$$

Since both sides are self-adjoint, (3.1) is obtained.

Q.E.D.

Lemma 3.1. If $\lambda_{\iota} \geqq 0, \Pi\left\{\lambda_{\iota}: \lambda_{\iota} \neq 0\right\}<+\infty,\left(\xi_{\iota}\right) \in S_{0}$ and $\sum \|\left|x_{\iota}\right| \xi_{\iota}-$ $\lambda_{\iota} \xi_{\iota} \|<+\infty$, then for any $0<\varepsilon<2^{-1}$ there exists a $J \subset \subset I$ such that for any $K \subset \subset I \backslash J$

$$
\left\|\underset{K}{\otimes}\left|x_{\iota}\right| \xi_{\iota}-\underset{K}{\otimes} \lambda_{\iota} \xi_{\iota}\right\|<\varepsilon
$$

Proof. Since $\lambda_{\iota} \geqq 0, \Pi\left\{\lambda_{\iota}: \lambda_{\iota} \neq 0\right\}<+\infty$ and $\left(\xi_{\iota}\right) \in S_{0}$, there is a $\mu>1$ with $\Pi_{J}\left\|\lambda_{\iota} \xi_{\iota}\right\|<\mu$ for $J \subset \subset I$. Choose any $0<\varepsilon<2^{-1}$. Since $\sum \|\left|x_{\iota}\right| \xi_{\text {، }}$ $-\lambda_{\iota} \xi_{\iota} \|<+\infty$, there exists a $J \subset \subset I$ such that for any $K \subset \subset I \backslash J$

$$
\sum_{K}\left\|\left|x_{\iota}\right| \xi_{\iota}-\lambda_{\iota} \xi_{\iota}\right\|<(2 \mu)^{-1} \varepsilon
$$

which implies 


$$
\begin{aligned}
& \left\|\underset{K}{\otimes}\left|x_{\iota}\right| \xi_{\iota}-\underset{K}{\otimes} \lambda_{\iota} \xi_{\iota}\right\| \\
& =\left\|\underset{\kappa}{\otimes}\left\{\lambda_{\iota} \xi_{\iota}+\left(\left|x_{\imath}\right| \xi_{\iota}-\lambda_{\imath} \xi_{\iota}\right)\right\}-\underset{K}{\otimes} \lambda_{\iota} \xi_{\iota}\right\| \\
& =\| \sum_{\iota \in K}\left(\left|x_{\iota}\right| \xi_{\iota}-\lambda_{\iota} \xi_{\iota}\right) \otimes\left(\underset{\substack{\kappa \in K \\
\kappa \neq \iota}}{\otimes} \lambda_{k} \xi_{\kappa}\right) \\
& +\sum_{\substack{\iota, l^{\prime}, \in K \\
\iota \neq \iota^{\prime}}}\left(\left|x_{\iota}\right| \xi_{\iota}-\lambda_{\iota} \xi_{\iota}\right) \otimes\left(\left|x_{\iota}\right| \xi_{\iota^{\prime}}-\lambda_{\iota} \xi_{\iota^{\prime}}\right) \otimes\left(\underset{\substack{\kappa \in K \\
\kappa \neq \iota, \iota^{\prime}}}{\otimes} \lambda_{\kappa} \xi_{\kappa}\right) \\
& +\cdots+\underset{K}{\otimes}\left(\left|x_{\iota}\right| \xi_{\iota}-\lambda_{\iota} \xi_{\iota}\right) \|<\varepsilon .
\end{aligned}
$$

Q.E.D.

In the following we designate the spectrum and the point spectrum of a closed operator $x$ by $\sigma(x)$ and $\sigma_{p}(x)$, respectively.

Let $z=u|z|$ be the polar decomposition of $z$. Let $e$ be the spectral projection of $|z|$ corresponding to $\left[\lambda_{0}-\varepsilon, \lambda_{0}+\varepsilon\right]$ for any given $\varepsilon>0$. If $\lambda_{0} \in \sigma(|z|) \backslash \sigma_{p}(|z|)$, there exists a non zero vector $\xi$ such that $e \xi=\xi$, $u^{*} u \xi=\xi, z \xi \neq 0$, which implies

$$
\||z| \xi-\lambda \xi\|<\varepsilon\|\xi\| \text { and }\left\|\left|z^{*}\right| u \xi-\lambda u \xi\right\|<\varepsilon\|\xi\|
$$

whenever $\left|\lambda-\lambda_{0}\right|<\varepsilon$.

$\boldsymbol{R}_{+}^{*}$ denotes the set of all positive numbers. Theorem 1.1 in [7] is then restated as follows: Let $y_{n}, n \in \mathbf{N}$ and $y$ be invertible, positive and self-adjoint operators on a separable Hilbert space. Then the following conditions are equivalent when $n$ tends to $+\infty$ :

(i) $f\left(y_{n}\right)$ converges strongly to $f(y)$ for every $f \in C\left(\boldsymbol{R}_{+}^{*}\right)$ which vanishes at 0 and $+\infty$;

(ii) $f\left(y_{n}\right)$ converges strongly to $f(y)$ for every bounded $f \in C\left(\mathbb{R}_{+}^{*}\right)$; and

(iii) $y_{n}^{i t}$ converges strongly to $y^{i t}$ for all $t \in \mathbb{R}$.

Using this we have

Theorem 3.3. (i) Assume that $x_{\iota} \neq 0, x_{\iota}=u_{\iota}\left|x_{\iota}\right|$ is the polar decomposition, and there is a $\lambda_{\iota} \in \sigma\left(\left|x_{\iota}\right|\right)$ for each $\iota \in I$ such ihat $\Pi\left\{\lambda_{\iota}: \lambda_{\iota} \neq 0\right\}<$ $+\infty$ and $\left\{\iota \in I: \lambda_{\iota} \notin \sigma_{p}\left(\left|x_{\iota}\right|\right)\right\}$ is countable. If $\sum\left|\lambda_{\iota}-1\right|<+\infty$, then there exists a non zero reference pair $\left(\xi_{0_{\iota}}, \eta_{0_{\iota}}\right)$ of $\left(x_{\iota}\right)$ satisfying $u_{\iota}^{*} u_{\iota} \xi_{0_{\iota}}=$ $\xi_{0 \iota}, \eta_{0 \iota}=u_{\iota} \xi_{0 \iota}$ and 


$$
\sum\left\|\left|x_{\iota}\right| \xi_{0 \iota}-\lambda \iota \xi_{0 \iota}\right\|<+\infty
$$

(ii) If $\left(\xi_{0 \iota}, \eta_{0_{\iota}}\right)$ is a non-zero reference pair of $\left(x_{\iota}\right)$ with (3.2) for some $\lambda_{\iota} \geqq 0$, then

$$
\left(\otimes c^{\prime} c x_{\iota}\right) \xi=\lim _{J \subset \subset I} y_{J} \xi
$$

for any $\xi \in D\left(\otimes c^{\prime} c x_{\iota}\right)$, where $w_{\iota}$ is a partial isometry with the initial space $\left\{\lambda \xi_{0 \iota}: \lambda \in \mathbb{C}\right\}$ and the final space $\left\{\lambda u_{\iota} \xi_{0_{\iota}}: \lambda \in \mathbb{C}\right\} ; y_{\iota} \equiv x_{\iota}, \iota \in J$ and $y_{\kappa} \equiv \lambda_{\kappa} w_{\kappa}, \kappa \in I \backslash J$ for each $J \subset \subset I ; y_{J} \equiv \otimes c^{\prime} c y_{\iota}$.

(iii) Assume that $\mathscr{H}_{\iota}$ is separable and $x_{\iota}$ is invertible, positive and self-adjoint on $\mathscr{H}_{\iota}$. If $\left(\xi_{0_{\iota}}, \xi_{0_{\iota}}\right)$ is a non-zero reference pair of $\left(x_{\iota}\right)$ satisfying $\left(\xi_{0 \iota}\right) \in c$, then $\otimes^{c} x_{\iota}^{i t}$ is unitary on $\mathscr{H}_{c}$ and

$$
\left(\otimes^{c} x_{\iota}\right)^{i t}=\otimes^{c} x_{\iota}^{i t}
$$

for all $t \in \boldsymbol{R}$.

Proof. (i) Let $I_{p}=\left\{\iota \in I: \lambda_{\iota} \notin \sigma_{p}\left(\left|x_{\iota}\right|\right)\right\}$ and $I_{0}=\left\{\iota \in I: \lambda_{\iota}=0\right\}$. Since $I_{p}$ is countable, $I_{p}$ is identified with $N$. Let $e_{m}, m \in N$ be the spectral projection of $\left|x_{m}\right|$ corresponding to $\left\{\lambda \in \boldsymbol{R}_{+}^{*}:\left|\lambda-\lambda_{m}\right| \leqq \varepsilon^{m+1}\right\}$ for any fixed $0<\varepsilon<2^{-1}$. By the discussion preceding to this theorem there is a unit vector $\xi_{0 m}$ such that $e_{m} \xi_{0 m}=\xi_{0 m}, u_{m}^{*} u_{m} \xi_{0 m}=\xi_{0 m}, x_{m} \xi_{0 m} \neq 0$ and

$$
\sum_{I_{p}}\left\|\left|x_{\iota}\right| \xi_{0 \iota}-\lambda \iota \xi_{0 \iota}\right\|<+\infty \text {. }
$$

For $\iota \in I \backslash I_{p}$ there is a unit vector $\xi_{0 \iota}$ in $D\left(x_{\iota}\right)$ with $\left|x_{\iota}\right| \xi_{0 \iota}=\lambda_{\iota} \xi_{0 \iota}$. Therefore $\left(\xi_{0_{\iota}}\right) \in S_{0}$ and (3.2) is obtained. Putting $\eta_{0 \iota} \equiv u_{\iota} \xi_{0 \iota}$ for all $\iota \in I$, we have $\left(\eta_{0}\right) \in S$.

If $\sum\left|\lambda_{\iota}-1\right|<+\infty$, namely, if $I_{0} \subset \subset I$ and $\Pi\left\{\lambda_{\iota}: \iota \notin I_{0}\right\}>0$, then

$$
\sum\left|\left\|x_{\iota} \xi_{0 \iota}\right\|-1\right| \leqq \sum\left\|\left|x_{\iota}\right| \xi_{0 \iota}-\lambda_{\iota} \xi_{0 \iota}\right\|+\sum\left|\left\|\lambda_{\iota} \xi_{0 \iota}\right\|-1\right|<+\infty
$$

which implies $\left(x_{\iota} \xi_{0_{\iota}}\right) \in S$ and $\left(x_{\iota}^{*} \eta_{0_{\iota}}\right)=\left(\left|x_{\iota}\right| \xi_{0_{\iota}}\right) \in S$. Since $\sum\left|\lambda_{\iota}-1\right|<+\infty$, $\left(\lambda_{\iota} \xi_{0_{\iota}}\right) \in S, \quad\left(\bar{\lambda}_{\iota} \eta_{0_{\iota}}\right) \in S$ and $\left(\bar{\lambda}_{\iota} \eta_{0_{\iota}}\right) \sim\left(\eta_{0_{\iota}}\right)$. Since $\left(\left|x_{\iota}\right| \xi_{0_{\iota}}\right) \sim\left(\lambda_{\iota} \xi_{0_{\iota}}\right)$ by (3.2), we have $\left(x_{\iota} \xi_{0 \iota}\right) \sim\left(\bar{\lambda}_{\iota} \eta_{0 \iota}\right)$ and hence $\left(x_{\iota} \xi_{0 \iota}\right) \sim\left(\eta_{0 \iota}\right)$. Therefore $\left(\xi_{0 \iota}, \eta_{0 \iota}\right)$ is a non zero reference pair of $\left(x_{\iota}\right)$ with desired properties, if we replace $\xi_{0 \iota}$ with $\iota \in I_{0}$ by any vector satisfying $u_{\iota}^{*} u_{\iota} \xi_{0 \iota}=\xi_{0 \iota}$. 
(ii) We use the same notations $I_{p}, I_{0}$ and $e_{m}$ as above. From (3.2), if $\sum\left|\lambda_{\iota}-1\right|=+\infty$, then $\Pi\left\|x_{\iota} \xi_{\iota}\right\|=0$ for all $\otimes \xi_{\iota} \in \odot\left(D\left(x_{\iota}\right), \xi_{0 \iota}\right)$ and $y_{J}=0$. Thus (3.3) holds.

If $\sum\left|\lambda_{\iota}-1\right|<+\infty$, there is a $\delta$ in $\left(0,2^{-1}\right)$ such that $\delta<\Pi_{K} \lambda_{\iota}<$ $\delta^{-1}$ for any $K \subset I \backslash I_{0}$. Choose an $\varepsilon>0$ with $\varepsilon<\delta$. From the definition of $e_{m}$, we have $\left(\lambda_{m}-\varepsilon^{m+1}\right) e_{m} \leqq\left|x_{m}\right| e_{m}$. Since $0<\prod_{m \in I_{p} \backslash I_{0}}\left(\lambda_{m}-\varepsilon^{m+1}\right)<$ $+\infty$, there exists a $0<\mu<1$ such that $\mu<\prod_{K}\left(\lambda_{m}-\varepsilon^{m+1}\right)<\mu^{-1}$ for any $K \subset I_{p} \backslash I_{0}$. Since $e_{m} \xi_{0 m}=\xi_{0 m}$ for $m \in I_{p}$, if $K^{\prime} \subset I_{p} \backslash I_{0}$, then

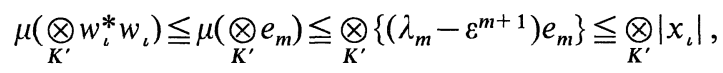

and if $K^{\prime \prime} \subset \boldsymbol{I} \backslash\left(I_{p} \cup I_{0}\right)$, then

$$
\delta\left(\bigotimes_{K^{\prime \prime}} w_{\iota}^{*} w_{\iota}\right) \leqq \bigotimes_{K^{\prime \prime}}^{\bigotimes}\left(\lambda_{\iota} w_{\iota}^{*} w_{\iota}\right) \leqq \underset{K^{\prime \prime}}{\bigotimes}\left|x_{\iota}\right|
$$

Since $\delta \mu<\min \{\delta, \mu\}<1$ and $\otimes_{I_{0}} \lambda_{\iota} w_{\iota}^{*} w_{\iota}=0$, we have

$$
(\delta \mu)^{2} y_{J}^{*} y_{J} \leqq\left(\otimes c^{\prime} c x_{\iota}\right)^{*}\left(\otimes c^{\prime} c x_{\iota}\right)
$$

on $D\left(\otimes{ }^{c^{\prime} c} x_{\iota}\right)$ for every $J \subset \subset I$. Since $\odot\left(D\left(x_{\iota}\right), \xi_{0_{\iota}}\right)$ is a core of $\otimes c^{c^{\prime} c} x_{\iota}$, there exists a sequence $\left\{\xi_{n}\right\}_{n=1}^{\infty}$ in $\odot\left(D\left(x_{\iota}\right), \xi_{0 \iota}\right)$ which converges to $\xi$ in the sense of the graph of $\otimes{ }^{c^{\prime} c} x_{\iota}$. It follows from (3.5) that $\left\{\xi_{n}\right\}_{n=1}^{\infty}$ is a Cauchy sequence in the sense of the graph of $y_{J}$. Therefore, since $y_{J}$ is closed, we have $\xi \in D\left(y_{J}\right)$. For the above $\varepsilon>0$ there exists an $n_{0}$ and a $J_{0} \subset \subset I$ such that for every $n \geqq n_{0}$ and for every $J \subset \subset I$ with $J_{0} \subset J$

$$
\left\|\left(\otimes c^{\prime} c x_{\iota}\right)\left(\xi_{n}-\xi\right)\right\|<\left(2+2(\delta \mu)^{-1}\right)^{-1} \varepsilon
$$

and

$$
\left\|\left(y_{J}-\otimes c^{c^{c}} x_{\iota}\right) \xi_{n_{0}}\right\|<2^{-1} \varepsilon
$$

Then we have

$$
\begin{aligned}
& \left\|\left(y_{J}-\otimes^{c^{\prime} c} x_{\iota}\right) \xi\right\| \\
& \quad \leqq\left\|y_{J}\left(\xi-\xi_{n_{0}}\right)\right\|+\left\|\left(y_{J}-\otimes^{c^{\prime} c} x_{\iota}\right) \xi_{n_{0}}\right\|+\left\|\left(\otimes c^{c^{\prime} c} x_{\iota}\right)\left(\xi_{n_{0}}-\xi\right)\right\| \\
& \quad \leqq\left(1+(\delta \mu)^{-1}\right)\left\|\left(\otimes{ }^{c^{\prime} c} x_{\iota}\right)\left(\xi_{n_{0}}-\xi\right)\right\|+\left\|\left(y_{J}-\otimes^{c^{\prime} c} x_{\iota}\right) \xi_{n_{0}}\right\|<\varepsilon
\end{aligned}
$$


for $\xi \in D\left(\otimes{ }^{c^{\prime} c} x_{\iota}\right)$.

(iii) Since $\left(\xi_{0_{\iota}}, \xi_{0_{\iota}}\right)$ is a non-zero reference pair of $\left(x_{\iota}\right)$, we have $\left(x_{\iota} \xi_{\iota}\right) \in S$ and $\left(x_{\iota} \xi_{\iota}\right) \sim\left(\xi_{\iota}\right)$ for all non zero $\otimes \xi_{\iota}$ in $\odot\left(D\left(x_{\iota}\right), \xi_{0}\right)$. Since $\left(x_{\iota} \xi_{\iota}\right) \in S$, there exists a $\lambda>1$ with $\prod_{K}\left\|x_{\iota} \xi_{\iota}\right\|<\lambda$ for all $K$. Since $\left(x_{\iota} \xi_{\iota}\right) \sim$ $\left(\xi_{\iota}\right)$, it follows from Lemma 3.3 in $[8]$ that for any $\varepsilon$ in $(0,1)$ there exists a $J_{0} \subset \subset I$ such that

$$
\left\|\underset{J}{\otimes} x_{\iota} \xi_{\iota}-\underset{J}{\otimes} \xi_{\iota}\right\|<\varepsilon / \lambda
$$

for all $J \subset I \backslash J_{0}$. Thus

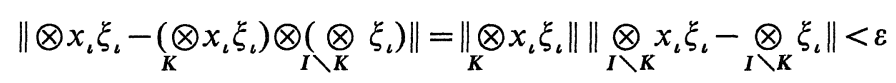

for all $K$ with $J_{0} \subset K \subset \subset I$.

Assume first that $I$ is countable. Let $I=N$ and $I_{n}=\{1, \ldots, n\}$. Denote $y \equiv \otimes^{c} x_{\iota}$ and $y_{n} \equiv y_{I_{n}} \quad\left(\right.$ or $y_{n} \equiv x_{I_{n}}$ ), where we take $\lambda_{\iota} w_{\iota}=1$. Since $y_{n}$ and $y$ are self-adjoint, $\left\|\left(y_{n}-i 1\right)^{-1}\right\| \leqq 1$ and $\left\|(y-i 1)^{-1}\right\| \leqq 1$. Let $C(x) \equiv(x+i 1)(x-i 1)^{-1}$. Let $D \equiv\left\{(y-i 1) \xi: \xi \in \odot\left(D\left(x_{\iota}\right), \xi_{0}\right)\right\}$. Since $\odot\left(D\left(x_{\iota}\right), \xi_{0_{\iota}}\right)$ is a core of $y$ by Theorem 1.1, $D$ is dense in $\mathscr{H}_{c}$. For any $\eta \in D$

$$
\begin{aligned}
& C\left(y_{n}\right) \eta-C(y) \eta \\
& \quad=\left(y_{n}+i 1\right)\left\{\left(y_{n}-i 1\right)^{-1}-(y-i 1)^{-1}\right\} \eta+\left(y_{n}-y\right)(y-i 1)^{-1} \eta \\
& \quad=\left(y_{n}+i 1\right)\left(y_{n}-i 1\right)^{-1}\left(y-y_{n}\right)(y-i 1)^{-1} \eta+\left(y_{n}-y\right)(y-i 1)^{-1} \eta .
\end{aligned}
$$

Since $\eta$ is of the form $(y-i 1) \xi$ for some $\xi \in \odot\left(D\left(x_{\iota}\right), \xi_{0_{\iota}}\right)$,

$$
\left\|C\left(y_{n}\right) \eta-C(y) \eta\right\| \leqq 2\left\|\left(y_{n}-y\right) \xi\right\|,
$$

which converges to 0 . Since $D$ is dense in $\mathscr{H}_{c}$ and since $C\left(y_{n}\right)$ and $C(y)$ are bounded, $C\left(y_{n}\right)$ converges strongly to $C(y)$. Since $\mathscr{H}_{c}$ is separable and since $y_{n}$ and $y$ are positive and self-adjoint, $f\left(y_{n}\right)$ converges strongly to $f(y)$ for every bounded function $f \in C\left(\boldsymbol{R}_{+}^{*}\right)$ by [7, Theorem 1.1]. Since $f(\lambda)=\lambda^{i t}$ for $\lambda \in \boldsymbol{R}_{+}^{*}$ and $t \in \boldsymbol{R}$ is a bounded continuous function in $\lambda$, it follows that

$$
\left(\otimes x^{c} x_{\imath}\right)^{i t} \xi=\lim _{n \rightarrow \infty}\left(\underset{I_{n}}{\otimes} x_{\imath}^{i t} \xi_{\iota}\right) \otimes\left(\underset{I \backslash I_{n}}{\otimes} \xi_{0 \iota}\right)
$$




$$
=\otimes x_{\imath}^{i t} \xi_{\iota}=\left(\otimes^{c} x_{\imath}^{i t}\right) \xi
$$

for any $\xi \in \odot\left(D\left(x_{\iota}\right), \xi_{0 \iota}\right)$ with $\xi=\otimes \xi_{\iota}$.

For a general $I$ we choose a countable $I_{0} \subset I$ such that $x_{\iota} \xi_{0 \iota}=\xi_{0 \iota}$ for $\iota \in I \backslash I_{0}$. Since $I_{0} \cup J$ is countable, we have

$$
\begin{aligned}
& \left(\otimes^{c} x_{\iota}\right)^{i t} \xi=\left(\left(\underset{I_{0} \cup J}{\otimes\left(\xi_{0}\right)} x_{\iota}\right)^{i t} \otimes\left(\underset{I \backslash\left(I_{0} \cup J\right)}{\otimes\left(\xi_{0}\right)} x_{\iota}\right)^{i t}\right) \xi \\
& =\left(\left(\underset{I_{0} \cup J}{\otimes\left(\xi_{0 c}\right)} x_{\iota}^{i t}\right) \otimes\left(\underset{N \backslash\left(I_{0} \cup J\right)}{\otimes\left(\xi_{0}\right)} x_{\iota}^{i t}\right)\right) \xi=\left(\otimes^{c} x_{\iota}^{i t}\right) \xi
\end{aligned}
$$

for any $\xi \in \odot\left(D\left(x_{\iota}\right), \xi_{0 \iota}\right)$ of the form $\xi=\xi_{J} \otimes\left(\otimes_{\backslash \backslash J} \xi_{0_{\iota}}\right)$ for some $J \subset \subset I$ and $\xi_{J} \in \otimes_{J} \mathscr{H}_{\imath}$. Thus we have (3.4).

Q.E.D.

Remark 3.2. If $\left(\xi_{0 \iota}, \eta_{0 \iota}\right)$ is a non-zero reference pair of $\left(x_{\iota}\right)$ with (3.2) and if $\sum\left|\lambda_{\iota}-1\right|<+\infty$, we have

$$
\left(\otimes c^{\prime} c x_{\iota}\right)=\lim _{J \subset \subset I} x_{J} \xi
$$

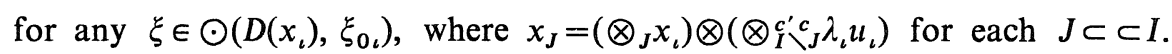

Remark 3.3. Assume the same assumption as the above (iii). Let $\left(\xi_{0 \iota}, \xi_{0_{\iota}}\right)$ be a non-zero reference pair of $\left(x_{\iota}\right)$ satisfying $\left(\xi_{0_{\iota}}\right) \in c$. Put $y_{\iota} \equiv \log x_{\iota}$ and $\pi_{\iota}^{c}\left(y_{\iota}\right) \equiv y_{\iota} \otimes\left(\otimes_{I \backslash\{\imath}^{c} 1_{\kappa}\right)$. Then

$$
\log \otimes{ }^{c} x_{\iota}=\sum \pi_{\iota}^{c}\left(y_{\iota}\right),
$$

where the sum of the right hand side is taken in the sense of Streit, [14].

Lemma 3.2. Let $z$ be a positive and self-adjoint operator. If $\varepsilon>0$ and $\|z \xi-\lambda \xi\| \leqq \varepsilon\|\xi\|$ for some non zero $\xi \in D(z)$, then there exists a $\lambda_{0} \in \sigma(z)$ such that $\left|\lambda-\lambda_{0}\right| \leqq \varepsilon$ and $\left\|z \xi-\lambda_{0} \xi\right\| \leqq 2 \varepsilon\|\xi\|$.

Proof. Let $e$ be the spectral projection of $z$ corresponding to $[\lambda-\varepsilon, \lambda+\varepsilon]$. Put $\xi_{0}=e \xi$. If $\xi_{0}=0$, then

$$
\|z \xi-\lambda \xi\|=\|(z-\lambda 1)(1-e) \xi\|>\varepsilon\|(1-e) \xi\|=\varepsilon\|\xi\|,
$$

which is impossible. Therefore $\xi_{0} \neq 0$. Hence $[\lambda-\varepsilon, \lambda+\varepsilon] \cap \sigma(z)$ is non empty and for any $\lambda_{0}$ in this intersection we have 


$$
\left\|z \xi-\lambda_{0} \xi\right\| \leqq\|z \xi-\lambda \xi\|+\left\|\left(\lambda-\lambda_{0}\right) \xi\right\| \leqq 2 \varepsilon\|\xi\|
$$

Q.E.D.

Corollary 3.1. If $x_{\iota} \neq 0$ for all $\iota \in I$ and if $\sum\left\|\left|x_{\iota}\right| \xi_{1 \iota}-\xi_{1 \iota}\right\|<+\infty$ for some $\left(\xi_{1 \iota}\right) \in S_{0}$, then there is a $\lambda_{\iota} \in \sigma\left(\left|x_{\iota}\right|\right)$ for each $\iota \in I$ such that $0<\Pi \lambda_{\iota}<+\infty$ and $\left\{\iota \in I: \lambda_{\iota} \notin \sigma_{p}\left(\left|x_{\iota}\right|\right)\right\}$ is countable.

Proof. By Lemma 3.2, there exists a $\lambda_{\iota} \in \sigma\left(\left|x_{\iota}\right|\right)$ such that $\left|1-\lambda_{\iota}\right| \leqq$ $\left\|\left|x_{\iota}\right| \xi_{1 \iota}-\xi_{1 \iota}\right\|$ and $\left\|\left|x_{\iota}\right| \xi_{1 \iota}-\lambda_{\iota} \xi_{1 \iota}\right\| \leqq 2\left\|\left|x_{\iota}\right| \xi_{1 \iota}-\xi_{1 \iota}\right\|\left\|\xi_{1 \iota}\right\|$. Then $\sum\left|1-\lambda_{\iota}\right|<$ $+\infty$ and $\sum\left\|\left|x_{\iota}\right| \xi_{1 \iota}-\lambda_{\iota} \xi_{1 \iota}\right\|<+\infty$. Except for a countable number of $\iota \in I$, we have $\left|x_{\iota}\right| \xi_{1 \iota}=\lambda_{\iota} \xi_{1}$.

Q.E.D.

Example 3.1. For $0<\varepsilon_{\iota}<1, \iota \in I$, put

$$
x_{\iota} \equiv\left(\begin{array}{cc}
1+\varepsilon_{\iota} & 0 \\
0 & 1-\varepsilon_{\iota}
\end{array}\right) \quad \text { and } \quad \xi_{\iota} \equiv\left(\begin{array}{c}
2^{-1 / 2} \\
2^{-1 / 2}
\end{array}\right) \text {. }
$$

If $\sum \varepsilon_{\iota}^{2}<+\infty$ and $\sum \varepsilon_{\iota}=+\infty$, then $\left(\xi_{\iota}\right) \in S_{0},\left(x_{\iota} \xi_{\iota}\right) \in S_{0},\left(x_{\iota}^{2} \xi_{\iota}\right) \in S_{0}$ and $\sum\left\|x_{\iota} \xi_{\iota}-\xi_{\imath}\right\|^{2}<+\infty$. Thus we have a situation where we have a nonzero reference pair $\left(\xi_{0 \iota}, \xi_{0_{\iota}}\right)$ of $\left(x_{\iota}\right)$ and yet there is no $\left\{\lambda_{\iota} \in \sigma\left(x_{\iota}\right): \iota \in I\right\}$ satisfying $0<\Pi \lambda_{\iota}<+\infty$.

For $\left(\xi_{\iota}\right)$ and $\left(\eta_{\iota}\right)$ in $S,\left(\xi_{\iota}\right)_{\widetilde{w}}\left(\eta_{\iota}\right)$ denotes the condition $\sum||\left(\xi_{\iota} \mid \eta_{\iota}\right)|-1|<$ $+\infty$, which is the weak equivalence due to von Neumann [11].

Remark 3.4. If $\left(\xi_{\iota}\right) \in S_{0},\left(\eta_{\iota}\right) \in S_{0}$ and $\sum\left\|\xi_{\iota}-\eta_{\iota}\right\|^{2}<+\infty$, then $\left(\xi_{\iota}\right)_{w}$ $\left(\eta_{\iota}\right)$.

Indeed, since $\left(\xi_{\iota}\right) \in S_{0}$ and $\left(\eta_{\iota}\right) \in S_{0}$, we have $\sup \left\|\xi_{\iota}\right\|<+\infty$ and sup $\left\|\eta_{\iota}\right\|<+\infty$, so that $\sum\left|\left\|\xi_{\iota}\right\|\left\|\eta_{\iota}\right\|-1\right|<+\infty$. Since $\sum\left\|\xi_{\iota}-\eta_{\iota}\right\|^{2}<+\infty$, we have $\sum\left|\operatorname{Re}\left(\xi_{\iota} \mid \eta_{\iota}\right)-1\right|<+\infty$. Therefore

$$
\begin{aligned}
& \sum\left\{\operatorname{Im}\left(\xi_{\iota} \mid \eta_{\iota}\right)\right\}^{2}=\sum\left\{\left|\left(\xi_{\iota} \mid \eta_{\iota}\right)\right|^{2}-\left|\operatorname{Re}\left(\xi_{\iota} \mid \eta_{\iota}\right)\right|^{2}\right\} \\
& \leqq \sum\left\{\left\|\xi_{\iota}\right\|^{2}\left\|\eta_{\iota}\right\|^{2}-\left|\operatorname{Re}\left(\xi_{\iota} \eta_{\iota}\right)\right|^{2}\right\} \\
& \leqq 2\left(\sup \left\|\xi_{\iota}\right\|\left\|\eta_{\iota}\right\|\right) \sum\left(\left|\left\|\xi_{\iota}\right\|\left\|\eta_{\iota}\right\|-1\right|+\left|\operatorname{Re}\left(\xi_{\iota} \mid \eta_{\iota}\right)-1\right|\right)<+\infty
\end{aligned}
$$

and there exists a $J \subset \subset I$ such that $2^{-1}<\operatorname{Re}\left(\xi_{\iota} \mid \eta_{\iota}\right)<2$ for $\iota \in I \backslash J$. Since 


$$
\begin{aligned}
\left|\left(\xi_{\iota} \mid \eta_{\iota}\right)\right| & =\operatorname{Re}\left(\xi_{\iota} \mid \eta_{\iota}\right)\left|1+\left\{\operatorname{Im}\left(\xi_{\iota} \mid \eta_{\iota}\right)\right\}^{2}\left\{\operatorname{Re}\left(\xi_{\iota} \mid \eta_{\iota}\right)\right\}^{-2}\right|^{1 / 2} \\
& \leqq \operatorname{Re}\left(\xi_{\iota} \mid \eta_{\iota}\right)+\left\{\operatorname{Im}\left(\xi_{\iota} \mid \eta_{\iota}\right)\right\}^{2}
\end{aligned}
$$

for all $\iota \in I \backslash J$, we have

$$
\begin{aligned}
& \sum||\left(\xi_{\iota} \mid \eta_{\iota}\right)|-1| \\
& \quad \leqq \sum||\left(\xi_{\iota} \mid \eta_{\iota}\right)\left|-\operatorname{Re}\left(\xi_{\iota} \mid \eta_{\iota}\right)\right|+\sum\left|\operatorname{Re}\left(\xi_{\iota} \mid \eta_{\iota}\right)-1\right|<+\infty
\end{aligned}
$$

Remark 3.5. Let $\left(\xi_{\iota}\right) \in S_{0}$ and $\left(\eta_{\iota}\right) \in S_{0}$. Define $\left(\xi_{\iota}\right)_{n}\left(\eta_{\iota}\right)$ for some fixed $n \geqq 1$ by $\sum\left\|\xi_{\iota}-\eta_{\iota}\right\|^{n}<+\infty$. Then " $\sim$ " is an equivalence relation. If $\left(\xi_{\iota}\right) \widetilde{\sim}\left(\eta_{\iota}\right)$, then $\left(\xi_{\iota}\right) \sim\left(\eta_{\iota}\right)$. If $\left(\xi_{\iota}\right) \sim\left(\eta_{\iota}\right)$, then $\left(\xi_{\iota}\right) \widetilde{\sim}\left(\eta_{\iota}\right)$. If $\left(\xi_{\iota}\right) \widetilde{2}\left(\eta_{\iota}\right)$, then $\left(\xi_{\iota}\right)_{\widetilde{w}}\left(\eta_{\iota}\right)$. In general, if $\left(\xi_{\iota}\right)_{n}\left(\eta_{\iota}\right)$ for $n \geqq 2$, then $\sum||\left(\xi_{\iota} \mid \eta_{\iota}\right)|-1|^{n / 2}<$ $+\infty$.

\section{§4. Modular Operator}

Let $\mathscr{H}_{\iota}$ denote the completion of a left Hilbert algebra $\mathscr{H}_{\iota}$, which is supposed to have a normalized idempotent element $\xi_{0 \iota}$ with $\xi_{0 \iota}^{\#}=\xi_{0 \iota}$.

Definition 4.1. An infinite tensor product of left Hilbert algebras $\mathfrak{H}_{\iota}$ is an involutive algebra of all $\otimes \xi_{\iota}$ in $\otimes \mathscr{H}_{\iota}$ with $\xi_{\iota} \in \mathfrak{A}_{\iota}$ and $\{\iota \in$ $\left.I: \xi_{\iota} \neq \xi_{0_{\iota}}\right\} \subset \subset I$ whose involution and product are defined by

$$
\left(\otimes \xi_{\iota}\right)^{\#}=\otimes \xi_{\iota}^{\#} \quad \text { and } \quad\left(\otimes \xi_{\iota}\right)\left(\otimes \eta_{\iota}\right)=\otimes \xi_{\iota} \eta_{\iota} .
$$

This is denoted by $\odot\left(\mathscr{U}_{\iota}, \xi_{0}\right)$.

Lemma 4.1. $\odot\left(\mathfrak{U}_{\iota}, \xi_{0_{\iota}}\right)$ is a left Hilbert algebra.

Proof. Let $\mathfrak{A}=\odot\left(\mathfrak{A}_{\iota}, \xi_{0_{\iota}}\right)$. Since $\xi_{0_{\iota}}=\xi_{0_{\iota}}^{\#}=\xi_{0 \iota}^{2}$, it follows that $(\xi \eta \mid \zeta)=\left(\eta \mid \xi^{\ddagger} \zeta\right)$ for $\xi, \eta$ and $\zeta$ in $\mathfrak{A}$ and that for each $\xi \in \mathfrak{A}$, the mapping: $\eta \in \mathfrak{A} \mapsto \mapsto \eta \eta \mathfrak{U}$ is continuous. Since $\mathfrak{A}_{\iota}^{2}$ is dense in $\mathfrak{A}_{\iota}$ and $\xi_{0 \iota}^{2}=$ $\xi_{0 \iota}, \mathfrak{A}^{2}$ is dense in $\mathfrak{A}$. Define $S_{\iota}$ and $S$ by $S_{\iota} \xi_{\iota}=\xi_{\iota}^{\#}$ for $\xi_{\iota} \in \mathfrak{A}_{\iota}$ and $S\left(\otimes{ }_{\iota} \xi\right)=\otimes S_{\iota} \xi_{\iota}$ for $\otimes \xi_{\iota} \in \mathfrak{A}$. Since $S_{\iota}$ is closable in $\mathscr{H}_{\iota}$ and $\xi_{0_{\iota}}=\xi_{0}^{\#}$, it follows that $\left(\xi_{0 \iota}, \xi_{0 \iota}\right)$ is a non-zero reference pair of $\bar{S}_{\iota}$. Therefore $S$ is closable by Lemma 2.4 .

Q.E.D. 
Remark 4.1. In order that $\odot\left(\mathfrak{A}_{\iota}, \xi_{0_{\iota}}\right)$ is a left Hilbert algebra, we have only to assume the existence of $\xi_{0 \iota} \in \mathfrak{A}_{\imath}$ for each $\iota \in I$ which satisfies that $\left(\xi_{0_{\iota}}\right) \in S_{0},\left(\xi_{0_{\iota}}^{2}\right) \in S_{0},\left(\xi_{0_{\iota}}\right) \sim\left(\xi_{0_{\iota} \iota}\right)$ and that $\left(\xi_{0_{\iota}}, \xi_{0_{\iota}}\right)$ is a non-zero reference pair of $\left(\bar{S}_{\iota}\right)$. In this case we can define $\otimes^{c} \bar{S}_{\iota}$ and $\otimes^{c} S_{\iota}^{*}$, which fulfill $\otimes^{c} S_{\iota}^{*} \bar{S}_{\iota}=\left(\otimes^{c} S_{\iota}^{*}\right)\left(\otimes{ }^{c} \bar{S}_{\iota}\right)$ for $c \equiv c\left(\xi_{0_{\iota}}\right)$.

It is clear from the definition that $\odot\left(\mathfrak{H}_{\iota}, \xi_{0_{\iota}}\right)$ is dense in $\otimes^{c} \mathscr{H}_{\iota}$. If we define a left representation $\pi$ of $\odot\left(\mathfrak{H}_{\iota}, \xi_{0 \iota}\right)$ on $\otimes^{c} \mathscr{H}_{\iota}$ by

$$
\pi\left(\otimes \xi_{\iota}\right) \otimes \eta_{\iota}=\otimes \xi_{\iota} \eta_{\iota},
$$

then $\pi\left(\odot\left(\mathfrak{H}_{\iota}, \xi_{0_{\iota}}\right)\right)^{\prime \prime}=\otimes^{c} \pi_{\iota}\left(\mathfrak{A}_{\iota}\right)^{\prime \prime}$, where $\pi_{\iota}$ is a left representation of $\mathfrak{A}_{\iota}$ on $\mathscr{H}_{\iota}$. This is proved by the similar argument as the proof of Corollary 3.3 in [9].

Let $\mathfrak{B}_{\iota}$ denote a Tomita algebra dense in $\mathscr{H}_{\text {}}$ with the modular automorphism $\Delta_{\iota}(z)$ for $\iota \in I$. If $\Delta_{\iota}(z) \xi_{0_{\iota}}=\xi_{0_{\iota}}$ for all $\iota \in I$ and $z \in \boldsymbol{C}$, we can define a modular automorphism $\Delta(z)$ on $\odot\left(\mathfrak{B}_{\iota}, \xi_{0_{\iota}}\right)$ by

$$
\Delta(z)\left(\otimes \xi_{\iota}\right)=\otimes \Delta_{\iota}(z) \xi_{\iota}
$$

for $\otimes \xi_{\iota}$ in $\odot\left(B_{\iota}, \xi_{0 \iota}\right)$. Here we denote by $\Delta_{\iota}$ the modular operator on $\mathscr{H}_{\iota}$ associated with the modular automorphism $\Delta_{\iota}(z), z \in \mathbb{C}$. Since $\left(\xi_{0_{\iota}}, \xi_{0_{\iota}}\right)$ is a non-zero reference pair of $\left(\Delta_{\iota}\right)$, we can define by Theorem 1.1 a positive self-adjoint operator $\Delta=\otimes^{c} \Delta_{\iota}$ in $\otimes^{c} \mathscr{H}_{\iota}$ for $c \equiv c\left(\xi_{0_{\iota}}\right)$. Here we suppose that $\mathscr{H}_{\iota}$ is separable for all $\iota \in I$. Since $\odot\left(\mathfrak{B}_{\iota}, \xi_{0_{\iota}}\right)$ is a core of $\otimes^{c} \Delta_{\iota}$, we have $\Delta^{i t}=\otimes^{c} \Delta_{\iota}^{i t}$ by Theorem 3.3. It then follows from the uniqueness of modular operator that $\Delta$ is the modular operator associated with $\Delta(z), z \in \boldsymbol{C}$.

Lemma 4.2. Suppose that $\mathscr{H}_{\imath}$ is separable for all $\iota \in I$. If $\Delta_{\iota}(z) \xi_{0 \text { 。 }}$ $=\xi_{0}$ for all $\iota \in I$ and $z \in \boldsymbol{C}, \odot\left(\mathfrak{B}_{\iota}, \xi_{0 \iota}\right)$ is a Tomita algebra and $\Delta=S^{*} \bar{S}$.

Proof. Since $S_{\iota}^{*} \xi_{0 \iota}=S_{\imath}^{*} \bar{S}_{\iota} \xi_{0 \iota}=\Delta_{\iota} \xi_{0 \iota}=\Delta_{\iota}(1) \xi_{0 \iota}=\xi_{0 \iota}$, we have $S^{*} \bar{S}\left(\otimes \xi_{\iota}\right)$ $=\otimes S_{\iota}^{*} \bar{S}_{\iota} \xi_{\iota}=\otimes \Delta_{\iota} \xi_{\iota}$ for $\otimes \xi_{\iota}$ in $\odot\left(\mathfrak{B}_{\iota}, \xi_{0_{\iota}}\right)$.

Corollary 4.1. Let $\omega_{\iota} \equiv \omega_{\xi_{0}}$, and $\omega \equiv \otimes \omega_{\iota}$ on $\otimes^{c} M_{\iota}$ for $c=c\left(\xi_{0}\right)$. If $\mathfrak{U}_{\iota}$ is separable for all $\iota \in I$, then $\sigma_{t}^{\omega}=\otimes{ }^{c} \sigma_{t}^{\omega} \iota$.

The separability assumption of $\mathfrak{A}_{\imath}$ in the above corollary will be 
omitted in Lemma 6.1.

\section{§. Infinite Product of $\sigma$-finite Measures}

We shall apply the results of $\S 3$ to the infinite product of $\sigma$-finite measure spaces and give a similar result as Hill's.

Throughout this section we assume the index set $I$ to be countably infinite.

Let $\left(\Omega_{\iota}, \mathscr{F}_{\iota}, v_{\iota}\right), \iota \in I$ be a probability space. Put $(\Omega, \mathscr{F})=\Pi\left(\Omega_{\iota}, \mathscr{F}_{\iota}\right)$, $v=\prod v_{\iota}, \mathscr{H}_{\iota}=L^{2}\left(\Omega_{\iota}, \mathscr{F}_{\iota}, v_{\iota}\right)$ and $Z_{\iota}=L^{\infty}\left(\Omega_{\iota}, \mathscr{F}_{\iota}, v_{\iota}\right)$. Then $(\Omega, \mathscr{F}, v)$ is a probability space. When a vector $\xi$ in $\mathscr{H}_{\iota}$ belongs to $Z_{\iota}$, we write the operator by $\pi_{\iota}(\xi)$. For an $\eta$ in $\mathscr{H}_{\iota}$ we denote by $\omega_{\eta}$ a measure on $\Omega_{\iota}$ or a positive linear form on $Z_{\iota}$ dfiened by $\omega_{\eta}(x)=(x \eta \mid \eta)$ for all $x \in Z_{\iota}$.

Let $\mu_{\iota}$ be a $\sigma$-finite measure on $\left(\Omega_{\iota}, \mathscr{F}_{\iota}\right)$ with $\mu_{\iota} \ll v_{\iota}$ and $h_{\iota}=d \mu_{\iota} / d v_{\iota}$. For $\xi_{\iota} \in D\left(h_{\iota}^{1 / 2}\right)$ with $h_{\iota}^{1 / 2} \xi_{\iota} \neq 0$, we define $\xi_{0 \iota}=\left\|\xi_{\iota}\right\|^{-1} \xi_{\iota}$ and $\eta_{0 \iota}=$ $\left\|h_{\iota}^{1 / 2} \xi_{\iota}\right\|^{-1} h_{\iota}^{1 / 2} \xi_{\iota}$. Then $\omega_{\eta_{0}}$ is a probability measure on $\Omega_{\iota}$ and $\omega_{\eta_{0} \iota} \ll v_{\iota}$. Therefore we can define a $\sigma$-finite measure $\mu_{J}$ for $J \subset \subset I$ on $\Omega$ by

$$
\mu_{J}=\left(\otimes_{J}\left\|h_{\iota}^{1 / 2} \xi_{\iota}\right\|^{-2} \mu_{\iota}\right) \otimes\left(\underset{I \backslash J}{\otimes} \omega_{\eta_{0} \iota}\right) .
$$

Then $\mu_{J}$ is a semi-finite normal trace on $\otimes^{c^{\prime}} Z_{\iota}$ for all $c^{\prime}$ with $c^{\prime} \tilde{u}$ $c\left(\eta_{0}\right)$.

Proposition 5.1. With the above notations, assume that $0<\Pi\left\|\pi\left(\xi_{\iota}\right)\right\|$ $<+\infty$ and $\mu_{\iota} \ll v_{\iota}$. If $\left(\eta_{0_{\iota}}, \eta_{0_{\iota}}\right)$ is a non-zero reference pair of $\left(\pi\left(\xi_{\iota}\right)\right)$, then $\mu \equiv \sup _{J \subset \subset I} \mu_{J}$ is a $\sigma$-finite measure on $\Omega$, which is singular to $\otimes \omega_{\eta_{\iota}}$ whenever $\left(\eta_{\iota}\right) \in S$ and $\left(\eta_{\iota}\right)_{u}\left(\eta_{0_{\iota}}\right)$. Moreover $\mu$ is a semi-finite normal trace on $\otimes c^{\prime} Z_{\iota}$ for all $c^{\prime}$ with $c^{\prime} \sim \underset{u}{c}\left(\eta_{0_{\iota}}\right)$.

Proof. If $\left(\eta_{0_{\iota}}, \eta_{0_{\iota}}\right)$ is a non zero reference pair of $\left(\pi\left(\xi_{\iota}\right)\right), \otimes^{c^{\prime}} \pi\left(\xi_{\iota}\right)$ is in $\otimes c^{\prime} B\left(\mathscr{H}_{\iota}\right)$ for $c^{\prime}=c\left(\eta_{0_{\iota}}\right)$. Since $0<\Pi\left\|\pi\left(\xi_{\iota}\right)\right\|<+\infty, \Pi_{I \backslash J}\left\|\pi\left(\xi_{\iota}\right)\right\|$ for $J \subset \subset I$ converges to 1 as $J$ tends to $I$. Since $\left\|h_{\iota}^{1 / 2} \xi_{\iota}\right\|^{2} \omega_{\eta_{0}} \leqq\left\|\pi\left(\xi_{\iota}\right)\right\|^{2} \mu_{\iota}$, $\left\{\left(\Pi_{I \backslash J}\left\|\pi\left(\xi_{\iota}\right)\right\|^{-2}\right) \mu_{J}: J \subset \subset I\right\} \quad$ is an increasing net of $\sigma$-finite measures on $\Omega$. Put

$$
\mu \equiv \lim _{J \subset \subset I}\left\{\left(\prod_{I \backslash J}\left\|\pi\left(\xi_{\iota}\right)\right\|^{-2}\right) \mu_{J}\right\}
$$


Then for $c^{\prime}=c\left(\eta_{0 \iota}\right)$

$$
\mu\left(\otimes c^{\prime}\left|\pi\left(\xi_{\iota}\right)\right|^{2}\right)=\sup _{J \subset \subset I} \prod_{I \backslash J}\left(\left\|\pi\left(\xi_{\iota}\right)\right\|^{-2}\left\|\pi\left(\xi_{\iota}\right) \eta_{0 \iota}\right\|^{2}\right)=1 .
$$

Since the set of $\left(\otimes_{J} x_{\iota}\right) \otimes\left(\otimes_{I \backslash J}^{c^{\prime}}\left\|\pi\left(\xi_{\iota}\right)\right\|^{-2} \pi\left(\xi_{\iota}\right)\right)$ for any $x_{\iota} \in Z_{\iota}$ and $J \subset \subset I$ is weakly total in $\otimes^{c^{\prime}} Z_{\iota}$, it follows that $\mu$ is semi-finite. Since each $Z_{\iota}$ is countably decomposable and $I$ is countable, $\otimes{ }^{c^{\prime}} Z_{\iota}$ is countably decomposable and hence $\mu$ is $\sigma$-finite.

If $\left(\eta_{\iota}\right) \in S_{0}$ and $\left(\eta_{\iota}\right) \underset{u}{\sim}\left(\eta_{0 \iota}\right)$, then the central carriers of $p_{c^{\prime}}$ for $c^{\prime} \equiv$ $c\left(\eta_{0_{\iota}}\right)$ and $p_{c^{\prime \prime}}$ for $c^{\prime \prime} \equiv c\left(\eta_{\iota}\right)$ in $\otimes Z_{\iota}$ are orthogonal by Theorem (2) in [1]. Therefore $\mu$ and $\otimes \omega_{\eta_{\iota}}$ are mutually singular.

Q.E.D.

Definition 5.1. Let $\mu_{\iota}$ be a $\sigma$-finite measure with $\mu_{\iota} \ll v_{\iota}$ and $h \equiv$ $d \mu_{\iota} / d v_{\iota}$. For $\xi_{\iota} \in D\left(h_{\iota}^{1 / 2}\right)$ with $0<\Pi\left\|\pi\left(\xi_{\iota}\right)\right\|<+\infty$ and $h_{\iota}^{1 / 2} \xi_{\iota} \neq 0$, let $\eta_{0 \iota} \equiv\left\|h_{\iota}^{1 / 2} \xi_{\iota}\right\|^{-1} h_{\iota}^{1 / 2} \xi_{\iota}$ and $\left(\eta_{0 \iota}, \eta_{0 \iota}\right)$ be a non-zero reference pair of $\left(\pi\left(\xi_{\iota}\right)\right)$. The $\sigma$-finite measure $\mu$ in Proposition 5.1 is denoted by $\mu^{\left(\xi_{c}\right)}$, since it depends on $\left(\xi_{\iota}\right) \in S_{0}$.

Theorem 5.1. Let $v_{\iota}, v, \mu_{\iota}, h_{\iota}$ be as before and let $\mu_{\iota} \sim v_{\iota}$ (resp. $\mu_{\iota} \ll$ $\left.v_{\iota}\right)$. Assume that $\xi_{\iota} \in D\left(h_{\iota}^{1 / 2}\right), \quad 0<\Pi\left\|\pi\left(\xi_{\iota}\right)\right\|<+\infty$ and $\left(\eta_{0_{\iota}}, \eta_{0_{\iota}}\right)$ is a non-zero reference pair of $\left(\pi\left(\xi_{\iota}\right)\right)$. Let $h_{0 \iota} \equiv\left\|\xi_{\iota}\right\|^{2}\left\|h_{\iota}^{1 / 2} \xi_{\iota}\right\|^{-2} h_{\iota}$ and $e_{\iota}$ be the spectral projection of $h_{0 \iota}^{1 / 2}$ corresponding to $\left[\lambda^{-1}, \lambda\right]$ for any fixed $\lambda>1$. Then the following nine conditions are equivalent for $c \equiv c\left(\xi_{0_{\iota}}\right)$ :

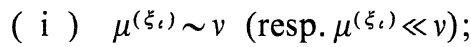

(ii ) $\left(\xi_{\iota}\right) \in S$ and $\left(\xi_{\iota}, \xi_{\iota}^{\prime}\right)$ is a non-zero reference pair of $\left(h_{0_{\iota}}^{1 / 2}\right)$;

(iii) $\left(\xi_{\iota}\right) \in S_{0}$ and $\left(\xi_{\iota}\right) \sim\left(h_{0}^{1 / 2} \xi_{\iota}\right)$;

(iv) $\left(\xi_{\iota}\right) \in S_{0}$ and $\left(\xi_{1 \iota}, \xi_{1 \iota}\right)$ is a non-zero reference pair of $\left(h_{0_{\iota}}^{1 / 2}\right)$ for some $\left(\xi_{1}\right) \in c$;

( v ) $\left(\xi_{\iota}\right) \in S,\left(e_{\iota} \xi_{2 \iota}\right) \in S,\left(h_{0 \iota}^{1 / 2} e_{\iota} \xi_{2 \iota}\right) \in S$ and $\left(e_{\iota} \xi_{2 \iota}\right) \sim\left(h_{0 \iota}^{1 / 2} e_{\iota} \xi_{2 \iota}\right)$ hold for some $\left(\xi_{2 \iota}\right) \in c$;

(vi) $\left(\xi_{\iota}\right) \in S,\left(e_{\iota} \eta_{\iota}\right) \in S,\left(h_{0}^{1 / 2} e_{\iota} \eta_{\iota}\right) \in S$ and $\left(e_{\iota} \eta_{\iota}\right) \sim\left(h_{0 \iota}^{1 / 2} \eta_{\iota}\right)$ hold for all $\left(\eta_{\iota}\right) \in c$ with $s\left(h_{\imath}\right) \eta_{\iota}=\eta_{\iota}$

(vii) $\left(\xi_{\iota}\right) \in S,\left(e_{\iota} \eta_{\iota}\right) \in S, \sum\left\|\log h_{0 \iota}^{1 / 2} e_{\iota} \eta_{\iota}\right\|^{2}<+\infty$ and $\sum\left|\left(\log h_{0 \iota}^{1 / 2} e_{\iota} \eta_{\iota} \mid \eta_{\iota}\right)\right|$ $<+\infty$ hold for all $\left(\eta_{\iota}\right) \in c$ with $s\left(h_{\imath}\right) \eta_{\iota}=\eta_{\iota}$;

(viii) $\left(\xi_{\iota}\right) \in S, \quad \xi_{3 \iota} \in D\left(h_{\iota}\right), \sum\left\|\log h_{0 \iota}^{1 / 2} \xi_{3 \iota}\right\|^{2}<+\infty$ and $\sum \mid\left(\log h_{0 \iota}^{1 / 2} \xi_{3 \iota} \mid\right.$ $\left.\xi_{3},\right) \mid<+\infty$ hold for some $\left(\xi_{3_{1}}\right) \in c$ with $s(h,) \eta_{\iota}=\eta_{1}$; and 
(ix) $\left(\xi_{\iota}\right) \in S$, and $\otimes{ }^{c} h_{0}^{i t}, t \in \boldsymbol{R}$ is strongly continuous one parameter unitary (resp.partial isometry) group.

Here $s\left(h_{\iota}\right)$ is a projection to $\left(\operatorname{Ker} h_{\imath}\right)^{\perp}$.

The proof of this theorem will be given after the following Proposition 5.2 .

Proposition 5.2. Under the same assumption as in Proposition 5.1 , let $\left(\eta_{0_{\iota}}, \eta_{0_{\iota}}\right)$ be a non-zero reference pair of $\left(\pi\left(\xi_{\iota}\right)\right)$. Then $\mu^{\left(\xi_{\iota}\right)} \sim v$ (resp. $\mu^{\left.\left(\xi_{\iota}\right) \ll v\right)}$ if and only if $\left(\xi_{\iota}\right) \in S_{0}$ and $\left(\xi_{0_{\iota}}\right) \sim\left(\eta_{0_{\iota}}\right)$. In this case $d \mu^{\left(\xi_{\iota}\right)} / d v=\left(\Pi\left\|\xi_{\iota}\right\|^{-2}\right) \otimes{ }^{c} h_{0 \iota}$ for $c=c\left(1_{\iota}\right)$ and $h_{0 \iota}=\left\|\xi_{\iota}\right\|^{2}\left\|h_{\iota}^{1 / 2} \xi_{\iota}\right\|^{-2} h_{\iota}$.

Proof. Suppose that $\left(\xi_{\iota}\right) \in S_{0}$ and $\left(\xi_{0 \iota}\right) \sim\left(\eta_{0}\right) . \quad\left(\xi_{\iota}\right) \in S_{0}$ implies $\left(\xi_{\iota}\right) \sim$ $\left(\xi_{0 \iota}\right)$ and hence $\left(\xi_{\iota}\right) \sim\left(\eta_{0_{\iota}}\right)$. Since $\left(\pi\left(\xi_{\iota}\right) \eta_{0_{\iota}}\right) \in S$ and $\left(\xi_{\iota}\right) \sim\left(\eta_{0_{\iota}}\right)$, we have $\left(\pi\left(\xi_{\iota}\right) \eta_{0_{\iota}}\right) \sim\left(1_{\iota}\right)$. It then follows that

$$
\left(\xi_{\iota}\right) \sim\left(\xi_{0_{\iota}}\right) \sim\left(\eta_{0_{\iota}}\right) \sim\left(\pi\left(\xi_{\iota}\right) \eta_{0_{\iota}}\right) \sim\left(1_{\iota}\right) .
$$

Since $\left(\xi_{0 \iota}\right) \sim\left(\eta_{0_{\iota}}\right),\left(\xi_{0 \iota}, \xi_{0 \iota}\right)$ is a non-zero reference pair of $\left(h_{0_{\iota}}^{1 / 2}\right)$ and $h \equiv \otimes^{c} h_{0_{\iota}}$ is obtained for $c=c\left(1_{\iota}\right)$. Let $\pi_{\iota}=L^{2}\left(\Omega_{\iota}, \mu_{\iota}\right) \cap L^{\infty}\left(\Omega_{\iota}, \mu_{\iota}\right)$ and $m_{\iota}$ be the linear span of $n_{\iota}^{*} n_{\iota}$. For any $\otimes^{c} x_{\iota}$ in $\otimes^{c} Z_{\iota}$ with $x_{\iota} \in \mathfrak{n}_{\iota}^{+}$ we have

$$
v\left(h\left(\left(\otimes_{J}^{\otimes} x_{\iota}\right) \otimes\left(\underset{I \backslash J}{\otimes} x_{\iota}\left|\pi\left(\xi_{\iota}\right)\right|^{2}\right)\right)\right)=\left(\Pi\left\|\xi_{\iota}\right\|^{2}\right) \mu_{J}\left(\otimes^{c} x_{\iota}\right)
$$

and hence

$$
\begin{aligned}
v\left(h\left(\otimes^{c} x_{\iota}\right)\right) & =\lim v\left(h\left(\left(\otimes_{J}^{\otimes} x_{\iota}\right) \otimes\left(\underset{I \backslash J}{\otimes c} x_{\iota}\left|\pi\left(\xi_{\iota}\right)\right|^{2}\right)\right)\right) \\
& =\lim \left(\Pi\left\|\xi_{\iota}\right\|^{2}\right) \mu_{J}\left(\otimes^{c} x_{\iota}\right)=\left(\Pi\left\|\xi_{\iota}\right\|^{2}\right) \mu^{\left(\xi_{\iota}\right)}\left(\otimes^{c} x_{\iota}\right) .
\end{aligned}
$$

Therefore $\mu^{\left(\xi_{\iota}\right) \ll v}$ and $d \mu^{\left(\xi_{\iota}\right)} / d v=\left(\Pi\left\|\xi_{\iota}\right\|^{-2}\right) \otimes c^{c} h_{0 \iota}$. If $\mu_{\iota} \sim v_{\iota}$, then $h_{0 \iota}$ is invertible and hence $d \mu^{\left(\xi_{c}\right)} / d v$ is also invertible or $\mu^{\left(\xi_{t}\right)} \sim v$.

Conversely, suppose that $\mu^{\left(\xi_{c}\right)} \ll v$. From Proposition 5.1 it follows that $\left(\eta_{0_{\iota}}\right) \widetilde{u}\left(1_{\iota}\right)$ or $\left(\eta_{0 \iota}\right) \sim\left(u_{\iota} 1_{\iota}\right)$ for some unitary $u_{\iota}$ in $Z_{\iota}^{\prime}=Z_{\iota}$ for each $\iota \in I$. Since $\left(\eta_{0_{\iota}}, \eta_{0_{\iota}}\right)$ is a non zero reference pair of $\left(\pi\left(\xi_{\iota}\right)^{*}\right)$ by Lemma 2.3, we have $\left(\pi\left(\xi_{\iota}\right) * \eta_{0}\right) \sim\left(u_{\iota} 1_{\iota}\right)$. Therefore $\left(\xi_{\iota}\right) \in S_{0}$ and $\left(\eta_{0}\right) \sim\left(u_{\iota} \xi_{\iota}\right)$. Since $\left(\xi_{\iota}\right) \sim\left(\xi_{0_{\iota}}\right)$, we have $\left(\eta_{0_{\iota}}\right) \sim\left(u_{\iota} \xi_{0_{\iota}}\right)$. Since $\left(\xi_{0_{\iota}}, u_{\iota} \xi_{0_{\iota}}\right)$ is a non zero reference pair of $\left(h_{0}\right)$, it follows from Theorem 1.1 that $\left(\xi_{0_{\iota}}\right) \sim\left(u_{\iota} \xi_{0_{\iota}}\right)$. 
Therefore $\left(\xi_{0_{\iota}}\right) \sim\left(\eta_{0_{\iota}}\right)$.

Q.E.D.

Proof of Theorem 5.1. (i) $\Rightarrow$ (ii). By Proposition $5.2\left(\xi_{0_{\iota}}, \xi_{0_{\iota}}\right)$ is a non-zero reference pair of $\left(h_{0}^{1 / 2}\right)$ and $\left(\xi_{\iota}\right) \in S_{0}$. It follows that $\left(\xi_{\iota}, \xi_{\iota}\right)$ is a non-zero reference pair of $\left(h_{0}^{1 / 2}\right)$.

(ii) $\Rightarrow$ (i). Put $\left(\xi_{0 \iota} \equiv\left\|\xi_{\iota}\right\|^{-1} \xi_{\iota}\right.$. Then $\left(\xi_{0 \iota}, \xi_{0 \iota}\right)$ is a non-zero reference pair of $\left(h_{0}^{1 / 2}\right)$. (i) follows from Proposition 5.2.

(ii) $\Leftrightarrow$ (iii) $\Rightarrow$ (iv). Clear.

(iv) $\Rightarrow$ (iii). Since $\left(\xi_{1 \iota}, \xi_{1 \iota}\right)$ is a non-zero reference pair of $\left(h_{0 \iota}^{1 / 2}\right)$, we have $\left(h_{0}^{1 / 2} \xi_{1 \iota}\right) \in S$ and $\left(\xi_{1 \iota}\right) \sim\left(h_{0 \iota}^{1 / 2} \xi_{1 \iota}\right)$. Since $c\left(\xi_{1 \iota}\right)=c\left(\xi_{0 \iota}\right)$, we have $\left(\xi_{1 \iota}\right) \sim\left(h_{0 \iota}^{1 / 2} \xi_{0_{\iota}}\right)$. Therefore $\left(\xi_{\iota}\right) \sim\left(\xi_{0_{\iota}}\right) \sim\left(\xi_{1 \iota}\right) \sim\left(h_{0 \iota}^{1 / 2} \xi_{0_{\iota}}\right) \sim\left(h_{0 \iota}^{1 / 2} \xi_{\iota}\right)$.

(iv) $\Leftrightarrow($ v) $\Leftrightarrow($ vi $) \Leftrightarrow($ vii $) \Leftrightarrow($ viii $) \Leftrightarrow($ ix $)$. By Theorem 3.1.

Remark 5.1. For each $J \subset \subset I$ a $\sigma$-finite measure $\mu(I \backslash J)=\left(\Pi_{J}\right.$ $\left.\left\|h_{\iota}^{1 / 2} \xi_{\iota}\right\|^{-2}\right) \mu^{\left(\xi_{\iota}: \iota \in I \backslash J\right)}$ on $\left(\prod_{\Gamma \backslash J} \Omega_{\iota}, \Pi_{\nwarrow \backslash J} \mathscr{F}_{\iota}\right)$ satisfies that $\mu^{\left(\xi_{\iota}\right)}=\left(\prod_{J} \mu_{\imath}\right) \times$ $\mu(I \backslash J)$. Therefore $\mu^{\left(\xi_{\iota}\right)}$ is a product measure of $\left\{\mu_{\iota}: \iota \in I\right\}$ in the sense of Hill. In Proposition 5.2, if we choose a measurable $\Omega_{\iota}^{\prime} \subset \Omega_{\iota}$ with $0<\mu_{\iota}\left(\Omega_{\iota}^{\prime}\right)<+\infty$ and define $\xi_{\iota}=\chi_{\Omega_{\iota}^{\prime}}$, then $0<\Pi\left\|\pi\left(\xi_{\iota}\right)\right\|<+\infty$ and $\left(\eta_{0 \iota}\right.$, $\left.\eta_{0_{\iota}}\right)$ is a non-zero reference pair of $\left(\pi\left(\xi_{\iota}\right)\right)$. Therefore $\mu^{\left(\xi_{\iota}\right)} \sim v$ if and only if $\left(\xi_{\iota}\right) \in S_{0}$ and $\left(\xi_{0 \iota}\right) \sim\left(\eta_{0_{\iota}}\right)$. This is a result of Hill. It should however be noted that we can not omitt the condition $\left(\xi_{\iota}\right) \in S_{0}$ as the following example shows.

Let $I \equiv \boldsymbol{N}$. Let $\Omega_{n} \equiv \boldsymbol{R}$ for $n \in I, v_{n}$ be a normal distribution with mean 0 and variance 1 , and $\mu_{n}$ be the Lebesgue measure. Put $\Omega_{n}^{\prime} \equiv$ $\left[-\lambda_{n}, \lambda_{n}\right], \lambda_{n}>0$ for all $n \in \mathbf{N}$ and $\xi_{n} \equiv \chi_{\Omega_{n}^{\prime}}$. Then

$$
\left(\xi_{0 n} \mid \eta_{0 n}\right)=\int_{-\lambda_{n} / \sqrt{2}}^{\lambda_{n} / \sqrt{2}} \exp \left(-\frac{x^{2}}{2}\right) d x\left\{\lambda_{n} \int_{-\lambda_{n}}^{\lambda_{n}} \exp \left(-\frac{x^{2}}{2}\right) d x\right\}^{-1 / 2} .
$$

By choosing $\lambda_{n}$ sufficiently small, we have $\left(\xi_{0_{\iota}}\right) \sim\left(\eta_{0_{\iota}}\right)$. However $\left(\xi_{\iota}\right) \notin S_{0}$ and hence $\left(\xi_{0}\right) \sim\left(1_{\iota}\right)$.

\section{§6. An Infinite Product of Semi-finite Weights}

Following the similar argument as the preceding section, we shall give a definition of an infinite tensor product of semi-finite faithful normal weights. $I$ is not necessarily countable. 
We begin by recalling the tensor product of semi-finite faithful normal weights $\psi_{1}$ on $\left(M_{1}\right)_{+}$and $\psi_{2}$ on $\left(M_{2}\right)_{+}$. Let $\mathfrak{A}_{j}$ denote the full left Hilbert algebra of $\left(M_{j}, \psi_{j}\right)$ obtained by the GNS construction for $j=1,2$. Let $\mathfrak{A}$ denote the full left Hilbert algebra formed from the algebraic tensor product of $\mathfrak{U}_{1}$ and $\mathfrak{U}_{2}$. If $\pi$ is the left representation of $\mathfrak{A}$, then $M_{1} \otimes M_{2}$ is isomorphic to $\pi(\mathfrak{A})^{\prime \prime}$. Through this isomorphism, the tensor product $\psi_{1} \otimes \psi_{2}$ of $\psi_{1}$ and $\psi_{2}$ is defined as the canonical weight of $\pi(\mathfrak{H})^{\prime \prime}$.

As Theorem 15.3 in [15] holds for a semi-finite faithful normal weight $\psi$ on $M_{+}$in place of a faithful normal positive linear form $\phi_{0}$ on $M$ by a slight improvement of the proof, we have that the necessary and sufficient condition for $a \psi a^{*} \leqq \psi, a \in \mathfrak{n}_{\psi}$ is that $\left\|\Delta_{\psi}^{-1 / 2} \pi_{\psi}(a) \Delta_{\psi}^{1 / 2}\right\|$ $\leqq 1, a \in \mathfrak{n}_{\psi}$. Here $\mathfrak{n}_{\psi}$ denote the set of all $x \in M$ with $\psi\left(x^{*} x\right)<+\infty$, $\pi_{\psi}$ the GNS representation of $M$ induced by $\psi$ and $\Delta_{\psi}$ the modular operator.

Let $\xi_{\text {c }}$ be a unit vector in $\mathscr{H}_{\text {}}$ which is cyclic and separating for $M_{\iota}$, and $\phi_{\iota} \equiv \omega_{\xi_{\iota}}$ on $M_{\iota}$. Let $\psi_{\iota}$ be a semi-finite faithful normal weight on $\left(M_{\iota}\right)_{+}$such that $\psi_{\iota}=h_{\iota}^{1 / 2} \phi_{\iota} h_{\iota}^{1 / 2}$ for some invertible, positive and self-adjoint operator $h_{\iota}$ affiliated with the centralizer $\left(M_{\iota}\right)_{\phi_{i}}$. Put $\boldsymbol{n}_{\iota} \equiv$ $\left\{x \in M_{\iota}: \psi_{\iota}\left(x^{*} x\right)<+\infty\right\}$. Let $e_{\iota}(n)$ denote the spectral projection of $h_{\iota}$ corresponding to $[0, n]$ for $n \in \boldsymbol{N}$. Let $J_{\xi}$, and $\Delta_{\xi}$, be a modular conjugation and a modular operator of $\left(M_{\imath}, \phi_{\iota}\right)$, respectively. Put $j_{\xi_{\iota}}(x)$ $\equiv J_{\xi_{\iota}} x J_{\xi_{\iota}}$ for $x \in M_{\iota}$. For each $x \in \mathfrak{n}_{\iota}$ we have

$$
\begin{aligned}
x h_{\iota}^{1 / 2} e_{\iota}(n) \xi_{\iota} & =x J_{\xi_{\iota}} \Delta \xi_{\iota}^{1 / 2} h_{\iota}^{1 / 2} e_{\iota}(n) \xi_{\iota}=x J_{\xi_{\iota}} h_{\iota}^{1 / 2} e_{\iota}(n) \dot{\xi}_{\iota} \\
& =x j_{\xi_{\iota}}\left(h_{\iota}^{1 / 2} e_{\iota}(n)\right) \xi_{\iota}=j_{\xi_{\iota}}\left(h_{\iota}^{1 / 2} e_{\iota}(n)\right) x \xi_{\iota} .
\end{aligned}
$$

Since $\left\{e_{\iota}(n+1)-e_{\iota}(n)\right\}_{n \in N}$ are orthogonal and since

$$
\sup _{n}\left\|j_{\xi_{\iota}}\left(h_{\iota}^{1 / 2} e_{\iota}(n)\right) x \xi_{\iota}\right\|^{2}=\sup _{n}\left\|x h_{\iota}^{1 / 2} e_{\iota}(n) \xi_{\iota}\right\|^{2} \leqq \psi_{\iota}\left(x^{*} x\right)<+\infty
$$

it follows that $\left\{x h_{\iota}^{1 / 2} e_{\iota}(n) \xi_{\iota}\right\}_{n=1}^{\infty}$ is a Cauchy sequence. We denote the limit $j_{\xi_{\iota}}\left(h_{\iota}^{1 / 2}\right) x \xi_{\iota}$ by $x h_{\iota}^{1 / 2} \xi_{\iota}$ symbolically.

For a fixed $x_{0 \iota} \in \mathfrak{n}_{\iota}$ with $x_{0 \iota} \neq 0$. put

$$
\xi_{0 \iota} \equiv\left\|x_{0_{\iota}} \xi_{\iota}\right\|^{-1} x_{0} \xi_{\iota} \text { and } \eta_{0_{\iota}} \equiv\left\|x_{0_{\iota}} h_{\iota}^{1 / 2} \xi_{\iota}\right\|^{-1} x_{0_{\iota}} h_{\iota}^{1 / 2} \xi_{\iota} \text {. }
$$


Define a semi-finite normal weight $\psi_{J}$ on $\left(\otimes c^{\prime} M_{\iota}\right)_{+}$for $c^{\prime}$ with $c^{\prime} \widetilde{p}$ $c\left(\eta_{0 l}\right)$ by

$$
\psi_{J}=\left(\otimes_{J}\left\|x_{0 \iota} h_{\iota}^{1 / 2} \xi_{\iota}\right\|^{-2} \psi_{\iota}\right) \otimes\left(\underset{I \backslash J}{\otimes} \omega_{\eta_{0} \iota}\right)
$$

Proposition 6.1. With the above notations, assume that $0<\Pi\left\|x_{0_{\iota}}\right\|<$ $+\infty$. If $\left(\eta_{0_{\iota}}, \eta_{0_{\iota}}\right)$ is a non-zero reference pair of $\left(x_{0_{\iota}}\right)$ and if $x_{0_{\iota}} \in \mathrm{n}_{\iota}$ with $\left\|\Delta_{\psi_{\iota}}^{1 / 2} \pi_{\psi_{\iota}}\left(x_{0 \iota}\right) \Delta_{\psi_{\iota}}^{1 / 2}\right\| \leqq\left\|x_{0 \iota}\right\|$, then $\psi=\lim _{J \subset c_{I}} \psi_{J}$ is a semi-finite faithful normal weight on $\left(\otimes c^{c^{\prime}} M_{\iota}\right)_{+}$for all $c^{\prime}$ with $c^{\prime} \underset{p}{c}\left(\eta_{0_{\iota}}\right)$.

Proof. Since $x_{0_{\iota}} \in n_{\iota}$ and $\left\|\Delta_{\psi_{\iota}}^{-1 / 2} \pi_{\psi_{\iota}}\left(x_{0 \iota}\right) \Delta_{\psi_{\iota}}^{1 / 2}\right\| \leqq\left\|x_{0_{\iota}}\right\|$, we have $x_{0 \iota} \psi_{\iota} x_{0_{\iota}}^{*} \leqq\left\|x_{0 \iota}\right\|^{2} \psi_{\iota}$ and hence $\left\|x_{0 \iota} h_{\iota}^{1 / 2} \xi_{\iota}\right\|^{2} \omega_{\eta_{0 \iota}} \leqq\left\|x_{0 \iota}\right\|^{2} \psi_{\iota}$. Therefore $\left\{\left(\prod_{I \backslash J}\left\|x_{0_{\iota}}\right\|^{-2}\right) \psi_{J}: J \subset \subset I\right\}$ is an increasing net of semi-finite normal weights on $\left(\otimes c^{\prime} M_{\iota}\right)_{+}$. Putting

$$
\psi \equiv \sup _{J \subset \subset I}\left\{\left(\prod_{I \backslash J}\left\|x_{0_{\iota}}\right\|^{-2}\right) \psi_{J}\right\}
$$

on $\left(\otimes c^{\prime} M_{\iota}\right)_{+}$, we know that $\psi$ is a normal weight on $\left(\otimes c^{c^{\prime}} M_{\iota}\right)_{+}$and that

$$
\psi\left(\otimes c^{\prime}\left|x_{0 \iota}\right|^{2}\right)=\sup _{J \subset \subset I} \prod_{I \backslash J}\left(\left\|x_{0 \iota}\right\|^{-2}\left\|x_{0 \iota} \eta_{0 \iota}\right\|^{2}\right)=1 .
$$

The semi-finiteness of $\psi$ is then proved by the similar way as Proposition 5.1. Let $S_{\iota}$ denote the carrier of $\omega_{\eta_{0}}$ in $M_{\iota}$ and $u_{\iota}$ be a partial isometry in $M_{\iota}^{\prime}$ such that $u_{\iota}^{*} u_{\iota} \eta_{0 \iota}=\eta_{0 \iota}$ and $c^{\prime}=c\left(u_{\iota} \eta_{0 \iota}\right)$. Since $S_{\iota} u_{\iota} \eta_{0 \iota}=$ $u_{\iota} \eta_{0_{\iota}}$ and since the carriers $\left(\otimes_{J} 1_{\iota}\right) \otimes\left(\otimes_{I \backslash J}^{c^{\prime}} S_{\iota}\right)$ of $\psi_{J}$ in $\otimes^{c^{\prime}} M_{\iota}$ are majorized by the carrier of $\psi$ for all $J \subset \subset I, \psi$ is faithful on $\otimes c^{c^{\prime}} M_{\iota}$.

Q.E.D.

Definition 6.1. The semi-finite faithful normal weight on $\left(\otimes c^{\prime} M_{\iota}\right)_{+}$ obtained in Proposition 6.1 is denoted by $\psi^{\left(x_{0} \iota\right)}$.

$\psi^{\left(x_{0}\right)}$ is considered as an infinite tensor product of normal weights $\psi_{\iota}$. We will show some conditions for $\psi^{\left(x_{0}\right)}$ to live on $\otimes^{c} M_{\iota}$ in Theorem 6.1 after the following proposition.

Proposition 6.2. Let $\xi_{\iota}, \phi_{\iota}, \psi_{\iota}, h_{\iota}$ and $x_{0 \iota}$ be as above. Let $\phi \equiv$ $\otimes \phi_{\iota}$ on $\otimes{ }^{c} M_{\iota}$ for $c \equiv c\left(\xi_{\iota}\right)$ and $\psi \equiv \psi^{\left(x_{0} \iota\right)}$ on $\otimes c^{\prime} M_{\iota}$ for some $c^{\prime} \sim c\left(\eta_{0}\right)$. Then 
(i) $\mathfrak{c}_{p} c^{\prime}$ if and only if $\left(x_{0 \iota} \xi_{\iota}\right) \in S_{0}$ and $\left(\xi_{0 \iota}\right) \underset{p}{\sim}\left(\eta_{0 \iota}\right)$;

and

(ii) under (i), $\psi=\psi \circ \sigma_{t}^{\phi}$ for all $t \in \mathbb{R}$.

Proof. (i) Suppose that $\left(x_{0 \iota} \xi_{\iota}\right) \in S_{0}$ and $\left(\xi_{0 \iota}\right) \widetilde{p}_{p}\left(\eta_{0 \iota}\right) . \quad\left(\xi_{0 \iota}\right) \sim_{p}\left(\eta_{0 \iota}\right)$ implies $\left(\xi_{0_{\iota}}\right) \sim\left(u_{\iota} \eta_{0_{\iota}}\right)$ for some partial isometry $u_{\iota}$ in $M_{\iota}^{\prime}$ with $u_{\iota}^{*} u_{\iota} \eta_{0_{\iota}}=$ $\eta_{0 \iota} . \quad\left(x_{0 \iota} \xi_{\iota}\right) \in S_{0}$ implies $\left(x_{0 \iota} \xi_{\iota}\right) \sim\left(\xi_{0 \iota}\right) \sim\left(u_{\iota} \eta_{0 \iota}\right)$. Since $\left(\eta_{0 \iota}, \eta_{0 \iota}\right)$ is a nonzero reference pair of $\left(x_{0_{\iota}}\right)$, we have $\left(x_{0_{\iota}}^{*} \eta_{0_{\iota}}\right) \sim\left(\eta_{0_{\iota}}\right)$. Since $0<\Pi\left\|x_{0_{\iota}}^{*}\right\|<$ $+\infty$, by Lemma 1 in [1] we have $\left(u_{\iota} x_{0 \iota}^{*} \eta_{0_{\iota}}\right) \in S$ and $\left(u_{\iota} x_{0 \iota}^{*} \eta_{0_{\iota}}\right) \sim\left(u_{\iota} \eta_{0_{\iota}}\right)$. Since $\left(x_{0} \xi_{\iota}\right) \sim\left(u_{\iota} \eta_{0 \iota}\right)$, we have $\left(\xi_{\iota}\right) \sim\left(u_{\iota} x_{0 \iota}^{*} \eta_{0 \iota}\right) \sim\left(u_{\iota} \eta_{0 \iota}\right)$ and hence $c \widetilde{p}$ $c\left(\eta_{0 \iota}\right) \underset{p}{\sim} c^{\prime}$.

Conversely, suppose that $c \widetilde{p}_{p} c^{\prime}$. Since $c^{\prime} \underset{p}{c} c\left(\eta_{0_{\iota}}\right)$, there exist partial isometries $u_{\iota}$ in $M_{\iota}^{\prime}$ so that $u_{\iota}^{*} u_{\iota} \eta_{0_{\iota}}=\eta_{0 \iota}$ and $\left(\xi_{\iota}\right) \sim\left(u_{\iota} \eta_{0_{\iota}}\right)$. Since $\left(\eta_{0_{\iota}}, \eta_{0_{\iota}}\right)$ is a non-zero reference pair of $\left(x_{0_{\iota}}\right)$, we have $\left(u_{\iota} x_{0_{\iota}}^{*} \eta_{0_{\iota}}\right) \sim\left(u_{\iota} \eta_{0_{\iota}}\right) \sim\left(\xi_{\iota}\right)$. Since $0<\Pi\left\|x_{0}\right\|<+\infty$, Lemma 1 in [1] implies that $\left(x_{0}, \xi_{\iota}\right) \in S$. Since $\xi_{\iota}$ is separating, $\left(x_{0 \iota} \xi_{\iota}\right) \in S_{0}$ and $\left(u_{\iota} \eta_{0 \iota}\right) \sim\left(x_{0}, \xi_{\iota}\right)$. Thus $\left(\xi_{0 \iota}\right) \sim\left(u_{\iota} \eta_{0_{\iota}}\right)$.

In order to prove (ii) we need to prepare the following lemma. Before going into the proof, we recall Theorem 14.4 in [16]. This is restated as follows: Let $\psi$ be a semi-finite faithful normal weight on $M_{+}$and $\sigma_{t}, t \in \boldsymbol{R}$ a one parameter group of *-automorphisms of $M$. If a weakly dense $*$-subalgebra $M_{0}$ of $M$ is invariant under $\sigma_{t}, t \in \mathbb{R}$ and if a pair of $\psi$ and $\sigma$ satisfies the KMS-condition for $M_{0}$, then $\sigma=\sigma^{\psi}$.

Lemma 6.1. Let $\phi \equiv \otimes \phi_{\iota}$ on $\otimes{ }^{c} M_{\iota}$ for $c \equiv c\left(\xi_{\iota}\right)$.

$$
\sigma_{t}^{\phi}\left(\otimes^{c} x_{\iota}\right)=\otimes{ }^{c} \sigma_{t}^{\phi}\left(x_{\iota}\right)
$$

for $\otimes^{c} x_{\iota}$ in $\otimes^{c} M_{\iota}$.

Proof. For any non zero $\otimes^{c} x_{\iota}$ in $\otimes^{c} M_{\iota}$, since $\left(\sigma_{t}^{\phi_{\iota}}\left(x_{\iota}\right) \xi_{\iota} \mid \xi_{\iota}\right)=$ $\left(x_{\iota} \xi_{\iota} \mid \xi_{\iota}\right)$ and $\left(x_{\iota} \xi_{\iota}\right) \sim\left(\xi_{\iota}\right)$, we can define a one parameter group of *automorphisms $\sigma_{t}=\otimes^{c} \sigma_{t}^{\phi}$ of $\otimes^{c} M_{\iota}$ by

$$
\sigma_{t}\left(\otimes{ }^{c} x_{\iota}\right)=\otimes^{c} \sigma_{t}^{\phi} \cdot\left(x_{\iota}\right)
$$

for $t \in \boldsymbol{R}$. Let $D$ denote the set of product vectors $\otimes \eta_{\iota}$ with $\{\iota \in I$ : 
$\left.\eta_{\iota} \neq \xi_{\iota}\right\} \subset \subset I$. Since $\left(\sigma_{t}(x) \xi \mid \eta\right)$ is continuous in $t \in \boldsymbol{R}$ for $\xi, \eta \in D$ and $x \in \otimes^{c} M_{\iota}$. Since $D$ is strongly total in $\mathscr{H}_{c}, \sigma_{t}$ is weakly continuous in $t \in \boldsymbol{R}$.

For any $x \equiv\left(\otimes_{J} x_{\iota}\right) \otimes\left(\otimes_{I \backslash J}^{c} 1_{\iota}\right)$ and $y \equiv\left(\otimes_{K} y_{\iota}\right) \otimes\left(\otimes_{I \backslash K}^{c} 1_{\iota}\right)$ in $\otimes^{c} M_{\iota}$, we have a bounded function $F_{\iota}(z)$ holomorphic in and continuous on $0 \leqq \operatorname{Im} z \leqq 1$ such that

$$
F_{\iota}(t)=\phi_{\iota}\left(\sigma_{t}^{\phi_{\iota}}\left(x_{\iota}\right) y_{\iota}\right) \text { and } F_{\iota}(t+i)=\phi_{\iota}\left(y_{\iota} \sigma_{t}^{\phi}\left(x_{\iota}\right)\right)
$$

for $t \in \boldsymbol{R}$. Therefore, by $\phi=\otimes \phi_{\iota}$, there is a bounded function $F(z)=$ $\prod_{J \cup K} F_{\iota}(z)$ holomorphic in and continuous on $0 \leqq \operatorname{Im} z \leqq 1$ such that

$$
F(t)=\phi\left(\sigma_{t}(x) y\right) \quad \text { and } \quad F(t+i)=\phi\left(y \sigma_{t}(x)\right) .
$$

Since the *-subalgebra of all finite linear combinations of $\left(\otimes_{J} x_{\iota}\right) \otimes$ $\left(\otimes \otimes_{I \backslash J} 1_{\iota}\right)$ with $x_{\iota} \in M$, and $J \subset \subset I$ is weakly dense in $\otimes^{c} M_{\iota}$ and is invariant under $\sigma_{t}, t \in \boldsymbol{R}$, it follows from the discussion preceding to this lemma that $\sigma_{t}=\sigma_{t}^{\phi}$ for all $t \in \boldsymbol{R}$.

Q.E.D.

Proof of (ii) in Proposition 6.2. Since $\psi_{\iota}=\psi_{\iota} \circ \sigma_{t}^{\phi}$, for any $x \in \otimes^{c} M_{\iota}$ of the form $\left(\otimes_{K} x_{\iota}\right) \otimes\left(\otimes_{I \backslash K}^{c} 1_{\imath}\right)$ we have

$$
\begin{aligned}
\left(\psi \circ \sigma_{t}^{\phi}\right)(x) & =\psi\left(\otimes \otimes^{c} \sigma_{t}^{\phi}\left(x_{\iota}\right)\right) \\
& =\lim _{K \subset J \subset \subset I} \prod_{J}\left\|x_{0 \iota} h_{\iota}^{1 / 2} \xi_{\iota}\right\|^{-2} \psi_{\iota}\left(\sigma_{t}^{\phi}\left(x_{\iota}\right)\right) \\
& =\lim _{K \subset J \subset \subset I} \prod_{J}\left\|x_{0 \iota} h_{\iota}^{1 / 2} \xi_{\iota}\right\|^{-2} \psi_{\iota}\left(x_{\iota}\right) \\
& =\psi\left(\otimes^{c} x_{\iota}\right)=\psi(x) .
\end{aligned}
$$

Q.E.D.

Theorem 6.1. Let $\xi_{\iota}, \phi_{\iota}, \psi_{\iota}, h_{\iota}$ and $x_{0_{\iota}}$ as before. Let $\phi \equiv \otimes \phi_{\iota}$ on $\otimes^{c} M_{\iota}$ for $c \equiv c\left(\xi_{\iota}\right)$ and $\psi \equiv \psi^{\left(x_{0}\right)}$ on $\otimes c^{\prime} M_{\iota}$ for $c^{\prime}{ }_{p}^{c}\left(\eta_{0 \iota}\right)$.

(1) Let $\lambda_{\iota} \equiv\left\|x_{0} \xi_{\iota}\right\|\left\|x_{0 \iota} h_{\iota}^{1 / 2} \xi_{\iota}\right\|^{-1}, h_{0 \iota} \equiv \lambda_{\iota}^{2} h_{\iota}$ and $e_{\iota}$ a spectral projection of $h_{0 \iota}^{1 / 2}$ corresponding to $\left[\lambda^{-1}, \lambda\right]$ for any fixed $\lambda>1$. It is sufficient for $\psi$ to be a semi-finite faithful normal weight on $\left(\otimes^{c} M_{\iota}\right)_{+}$ that one of the following conditions holds:

(i) $\left(x_{0 \iota} \xi_{\iota}\right) \in S_{0}$ and $\left(\xi_{0 \iota}\right) \underset{p}{\sim}\left(\eta_{0 \iota}\right)$; 
(ii) $\left(\xi_{1 \iota}, \xi_{1 \iota}\right)$ is a non-zero reference pair of $\left(h_{0 \iota}^{1 / 2}\right)$ for some $\left(\xi_{1},\right) \in c$;

(iii) $\left(e_{\iota} \xi_{\iota}\right) \in S,\left(h_{0}^{1 / 2} e_{\iota} \xi_{\iota}\right) \in S$ and $\left(e_{\iota} \xi_{\iota}\right) \sim\left(h_{0 \iota}^{1 / 2} e_{\iota} \xi_{\iota}\right)$;

(iv) $\left(e_{\iota} \xi_{\iota}\right) \in S, \sum\left\|\log h_{0}^{1 / 2} e_{\iota} \xi_{\iota}\right\|^{2}<+\infty$ and $\sum\left|\left(\log h_{0 \iota}^{1 / 2} e_{\iota} \xi_{\iota} \mid \xi_{\iota}\right)\right|<+\infty$; and

(v) $\otimes^{c} h_{0_{\iota}}^{i t}, t \in \boldsymbol{R}$ is a strongly continuous one parameter unitary group.

Under conditions from (ii) to (v) $h \equiv\left(\prod\left\|x_{0} \xi_{\iota}\right\|^{-2}\right) \| \otimes{ }^{c} h_{0}$ is affiliated with $\left(\otimes^{c} M_{\iota}\right)_{\phi}$ and $\psi=\phi \circ h$. In particular, if $x_{0 \iota} \in M_{\psi \iota}$, then $\sigma_{t}^{\psi}=$ $\otimes{ }^{c} \sigma_{t}^{\psi}$.

(2) If $x_{0 \iota}$ commutes with $h_{\iota}$ for all $\iota \in I$, every condition in (1) is necessary for $\psi$ to be a semi-finite faithful normal weight on $\left(\otimes{ }^{c} M_{\imath}\right)_{+}$.

Proof. (1) By Proposition 6.2, (i) is a sufficient condition.

If one of the conditions from (ii) to (v) holds, we can define $\otimes^{c} h_{0_{\iota}}^{1 / 2}$ by Theorem 3.1 and $\otimes^{c} h_{0 \iota}=\left(\otimes^{c} h_{0 \iota}^{1 / 2}\right)^{2}$ by Theorem 3.2. We have for all non zero $\otimes^{c} x_{\iota}$ in $\left(\mathrm{m}_{\psi}\right)_{+}$,

$$
\begin{aligned}
\psi\left(\otimes^{c} x_{\iota}\right) & =\sup \left\{\left(\prod_{I J}\left\|x_{0_{\iota}}\right\|^{-2}\right) \psi_{J}\left(\otimes^{c} x_{\iota}\right)\right\} \\
& =\lim \prod_{J}\left\{\left\|x_{0_{\iota}} h_{\iota}^{1 / 2} \xi_{\iota}\right\|^{-2} \psi_{\iota}\left(x_{\iota}\right)\right\} \\
& =\lim \prod_{J}\left\{\left\|x_{0_{\iota}} \xi_{\iota}\right\|^{-2} \phi_{\iota}\left(h_{0_{\iota}} x_{\iota}\right)\right\} \\
& =\phi\left(h \otimes{ }^{c} x_{\iota}\right) .
\end{aligned}
$$

From (ii) of Proposition 6.2, we know that $h$ is affiliated with $\left(\otimes^{c} M_{\imath}\right)_{\phi}$ and $\psi=\phi \circ h$. Since $h$ is invertible, $\psi$ is faithful.

Suppose that $x_{0 \iota}$ is in $\left(M_{\imath}\right)_{\psi_{\iota}}$. By virtue of Lemma 6.1 we have $\sigma_{t}^{\phi}=\otimes^{c} \sigma_{t}^{\phi}$. Define a $*$-automorphism $\sigma_{t}$ of $\otimes^{c} M_{\iota}$ by

$$
\sigma_{t}(x)=\left(\otimes^{c} h_{0 \iota}^{i t}\right) \sigma_{t}^{\phi}(x)\left(\otimes^{c} h_{0 \iota}^{-i t}\right)
$$

for $x \in \otimes \otimes^{c} M_{\iota}$. Since $\sigma_{t}^{\psi} \iota(y)=h_{0 \iota}^{i t} \sigma_{t}^{\phi}(y) h_{0 \iota}^{-i t}$ for $y \in M_{\iota}$, we have

$$
\sigma_{t}\left(\otimes{ }^{c} x_{\iota}\right)=\otimes^{c} \sigma_{t}^{\psi} \cdot\left(x_{\iota}\right), \quad t \in \boldsymbol{R}
$$

and $\sigma_{t}$ is weakly continuous by Theorem 3.1. For any $x \equiv\left(\otimes_{J} x_{\imath}\right) \otimes$ 
$\left(\otimes_{I \backslash J}^{c}\left|x_{0 \iota}\right|\right)$ and $y \equiv\left(\otimes_{K} y_{\iota}\right) \otimes\left(\otimes_{I \backslash K}\left|x_{0 \iota}\right|\right)$ with $x_{\iota}$ and $y_{\iota}$ in $\mathfrak{n}_{\psi_{\iota}}$ we have a bounded function $F_{\imath}(z)$ holomorphic in and continuous on $0 \leqq \operatorname{Im} z \leqq 1$ such that

$$
F_{\iota}(t)=\dot{\psi}_{\iota}\left(\sigma_{t}^{\psi} \iota\left(x_{\iota}\right) y_{\iota}\right) \quad \text { and } \quad F_{\iota}(t+i)=\dot{\psi}_{\iota}\left(y_{\iota} \sigma_{t}^{\psi} \iota\left(x_{\iota}\right)\right)
$$

for $t \in \boldsymbol{R}$, where $\dot{\psi}_{\iota}$ is the linear extension of $\psi_{\iota}$ to $\mathrm{m}_{\psi_{\iota}}$. Therefore, since $x_{0 \iota} \in\left(M_{\iota}\right)_{\psi \iota}$ and $c^{\prime} \underset{p}{\sim} c\left(\eta_{0 \iota}\right)$, there is a bounded function

$$
F(z)=\prod_{J \cup K} \psi_{\iota}\left(\left|x_{0}\right|^{2}\right)^{-1} F_{\iota}(z)
$$

holomorphic in and continuous on $0 \leqq \operatorname{Im} z \leqq 1$ such that

$$
F(t)=\dot{\psi}\left(\sigma_{t}(x) y\right) \quad \text { and } \quad F(t+i)=\dot{\psi}\left(y \sigma_{t}(x)\right)
$$

Thus $\sigma_{t}=\sigma_{t}^{\psi}$ and hence $\sigma_{t}^{\psi}=\otimes^{c} \sigma_{t}^{\psi}$, for all $t \in \boldsymbol{R}$.

(2) By means of the proof of necessity of (i) in Proposition 6.2, we have $\left(x_{0 \iota} \xi_{\iota}\right) \sim\left(\xi_{0 \iota}\right) \sim\left(u_{\iota} \eta_{0 \iota}\right)$ for some unitary $u_{\iota}$ in $M_{\iota}^{\prime}$. Then $\left(\xi_{0 \iota}\right.$, $\left.u_{\iota}^{*} \xi_{0 \iota}\right)$ is a non-zero reference pair of $\left(h_{0 \iota}^{1 / 2}\right)$. Hence by Theorem 3.1 we have every condition in (1).

Q.E.D.

\section{Acknowledgements}

The earlier version of this paper was completed while the author was staying at Reseach Institute for Mathematical Sciences, in Kyoto University, from April until September, 1970. He would like to thank Professor H. Araki for his valuable discussion, warm hospitality at RIMS, taking the pains of reading the manuscript carefully and giving him valuable comments, improvements and corrections. He would also like to thank Professor S. Kitagawa for his discussion.

\section{References}

[1] Araki, H. and Nakagami, Y., A remark on an infinite tensor product of von Neumann algebras. Publ. RIMS, Kyoto Univ. 8 (1972), 363-374.

[2] Araki, H. and Woods, E. J., A classification of factors. Publ. RIMS, Kyoto Univ. 4 (1968), 51-130.

[3] Connes, A., Un nouvel invariant pour les algèbres de von Neumann. C. $R$. Acad. Sc. 273 (1971), 900-903. 
[4] Hamachi, T., Equivalent measures on product spaces. Mem. Fac. Sci. Kyushu Univ. 27 (1973), 335-341.

[5] Hill, D. G. B., $\sigma$-finite invariant measures on infinite product spaces. Trans. Amer. Math. Soc. 153 (1971), 347-370.

[6] Ikunishi, A. and Nakagami, Y., Automorphism groups of von Neumann algebras and semi-finiteness of an infinite tensor product of von Neumann algebras. To appear.

[7] Kallman, R. R., Groups of inner automorphisms of von Neumann algebras. J. Functional Analysis 7 (1971), 43-60.

[8] Nakagami, Y., Infinite tensor products of ron Neumann algebras, I. Kōdai Math. Sem. Rep. 22 (1970), 341-354.

[9] Nakagami, Y., Infinite tensor products of von Neumann algebras, II. Publ. RIMS, Kyoto Univ. 6 (1970), 257-292.

[10] Nakagami, Y., Infinite tensor products of operators. Sûrikaiseki Kenkyûsho Kôkyûroku 104 (1970), 109-117 (Japanese).

[11] von Neumann, J., On infinite direct products. Compositio Math. 6 (1937), $1-77$.

[12] Pedersen, G. K. and Takesaki, M., The Radon-Nikodym theorem for von Neumann algebras. Acta Math. 130 (1973), 53-87.

[13] Reed, M. C., On self-adjointness in infinite tensor product spaces. J. Functional Analysis 5 (1970), 94-124.

[14] Streit, L., Test function spaces for direct product representations of the canonical commutation relations. Commun. math. Phys. 4 (1967), 22-31.

[15] Takesaki, M., Tomita's theory of modular Hilbert algebras and its applications. SpringerVerlarg, 1970.

[16] Takesaki, M., The theory of operator algebras. Univ. California, Los Angeles, $1969 / 70$

Note added in proof. The separability assumption of $\mathscr{H}$, in (iii) of Theorem 3.3 can be omitted by using Remark 3.10 in the following paper:

Araki, H. and Woods, E.J., Topologies induced by representations of the canonical commutation relations. Reports on Math. Phys. 4 (1973), 227-254.

Therefore Lemma 4.2 and Corollary 4.1 hold without the separability assumption and Lemma 6.1 is clear from Corollary 4.1 . 
